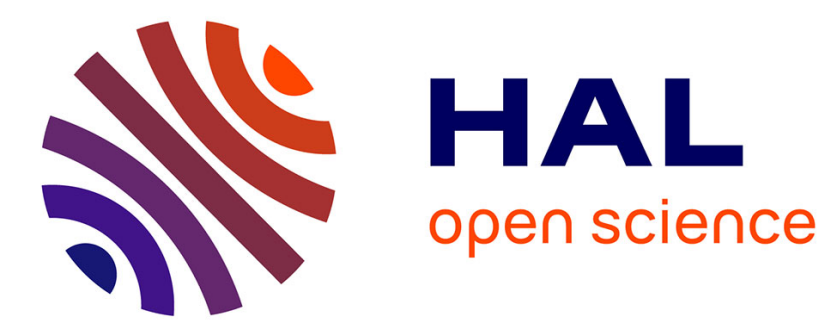

\title{
Treating volumetric inequality constraint in a continuum media with a coupled X-FEM/Level-Set strategy
}

\author{
Nicolas Bonfils, Nicolas Chevaugeon, Nicolas Moës
}

\section{To cite this version:}

Nicolas Bonfils, Nicolas Chevaugeon, Nicolas Moës. Treating volumetric inequality constraint in a continuum media with a coupled X-FEM/Level-Set strategy. Computer Methods in Applied Mechanics and Engineering, 2012, 205, pp.16-28. 10.1016/j.cma.2011.02.012 . hal-00569557

\section{HAL Id: hal-00569557 \\ https://hal.science/hal-00569557}

Submitted on 25 Feb 2011

HAL is a multi-disciplinary open access archive for the deposit and dissemination of scientific research documents, whether they are published or not. The documents may come from teaching and research institutions in France or abroad, or from public or private research centers.
L'archive ouverte pluridisciplinaire HAL, est destinée au dépôt et à la diffusion de documents scientifiques de niveau recherche, publiés ou non, émanant des établissements d'enseignement et de recherche français ou étrangers, des laboratoires publics ou privés. 


\title{
Treating volumetric inequality constraint in a continuum media with a coupled X-FEM/Level-Set strategy
}

\author{
N. Bonfils, N. Chevaugeon, N. Moës
}

\begin{abstract}
Some mechanical problems involve inequality kinematic constraint. This study deals with an original approach to handle those difficult problems. The main issue is the treatment of the variational inequalities due to the fact that the constrained area is a priori unknown. The method, introduced here, is to find the exact constrained area iteratively starting from an intial trial one. Thanks to numerical tools such as level-set and X-FEM we turn the constrained minimization problem into a shape equilibrium problem.
\end{abstract}

\section{Introduction}

The goal of this study is to propose a new approach to deal with continuum media subjected to a kinematic inequality constraint. A kinematic constraint is a restriction on the deformations a body can undergo. In this document, we will treat the problem of a media subjected to a volumetric inequality constraint. In fact, this paper is first step toward handling an extensibility limit constraint in hyperelastic materials.

Classical methods used to enforce inequality constraint within a media are the Lagrange multiplier method [2], the penalty method [7], and the augmented Lagrangian method [3]. The penalty method does not permit to enforce exactly the kinematic constraint and can lead to a bad conditioning of the tangent stiffness matrix. Both the Lagrangian and augmented Lagrangian method assure the respect of the constraint. However, their numerical treatment imply the use of specific algorithms such as the Uzawa method [2] or the Generalized Newton Method [8], and parameters have to be specified. These parameters play a major role in the convergence of those algorithms and it is often very difficult to choose them appropriately. Note that in most cases, those algorithms are used to treat inequality constraint on the boundary of a media (unilateral contact condition). The constraint studied here is a volumetric constraint.

After a brief statement of the constraint minimization problem studied here, we will describe in the first section an already existing method [6] (close to augmented lagrangian method) used for contact problems and that can be applied to our problem. This is an interesting method since it does not need the use of a Generalized Newton Method and can be solved using a kind of Active Set algorithm [9]. However, we will show that in our particular case, this method is not really efficient. First, it offers poor rate of convergence and the presence of user-set parameters for the active set algorithm may deteriorate its stability.

In the next section, we will present our new approach. The strategy consists in turning the minimization problem under inequality constraint into a shape equilibrium one. The inequality constraint induces variational inequalities because we do not know a priori the shape of the active constraint and unactive constraint zone in the media. The strategy proposed here is to replace the inequality constraint by an equality constraint over an unknown domain. This domain is called the active constraint domain. Hence, we assimilate the media to a two elastic phases media. On one phase, no constraints are imposed and on the second one an equality constraint is imposed. Each phase has a mechanical behaviour that depends on the state of the constraint (active or inactive). With the help of the configurational mechanic and the notion of configurational force, the exact 
shape of each phase is found iteratively. Finally, the notion of level-set [23] and the X-FEM [24, 5] will be used to give an implicit description of the interface separating the two phases. Several numerical tests will be shown in the final section so as to compare the first method to the new one.

\section{Statement of the problem}

A linear elasticity two-dimensional problem is considered, with an isotropic homogeneous material in a plane strain state. Consider the domain $\Omega$ as shown in Figure 1. On the boundary, a Dirichlet condition $\underline{u}=\underline{u}_{d}$ is applied on $\Gamma_{u}$ and a Neumann condition $\underline{\underline{\sigma}} \cdot \underline{n}=\underline{t} d$ is prescribed on $\Gamma_{t}$. Finally, the whole domain is submited to a body force $f$.

In this study we will adopt a particular form of the constraint in the small strain setting. The constraint reads:

$$
\gamma(\underline{\underline{\varepsilon}})=\operatorname{Tr} \underline{\underline{\varepsilon}}-\alpha \leq 0 \text { on } \Omega
$$

with $\underline{\underline{\varepsilon}}$, the classical strain tensor and $\operatorname{Tr} \underline{\underline{\varepsilon}}$ the trace of $\underline{\underline{\varepsilon}}$.

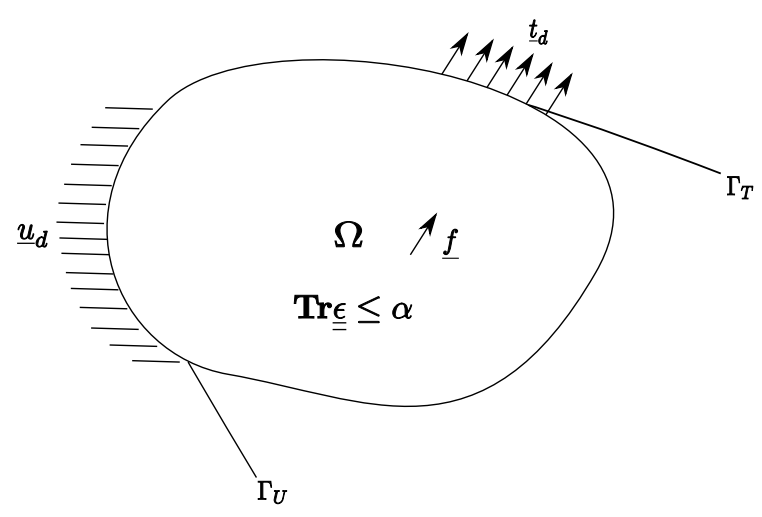

Figure 1: Problem statement

Let use define the space of kinematically admissible displacements

$$
\begin{aligned}
& V=\left\{\underline{v} \in H^{1}(\Omega) \mid \underline{v}=\underline{u}_{d} \text { on } \Gamma_{U}\right\} \\
& V^{0}=\left\{\underline{v} \in H^{1}(\Omega) \mid \underline{v}=0 \text { on } \Gamma_{U}\right\}
\end{aligned}
$$

And the total potential energy of the mechanical system:

$$
\Pi(\underline{u})=\int_{\Omega} \psi(\underline{\underline{\varepsilon}}) d \Omega-\int_{\Omega} \underline{f} \cdot \underline{u} d \Omega-\int_{\Gamma_{t}} \underline{t}_{d} \cdot \underline{u} d \Gamma
$$

where $\psi(\underline{\underline{\varepsilon}})=\frac{1}{2} \underline{\underline{\varepsilon}}: \underline{\underline{\underline{C}}}: \underline{\underline{\varepsilon}}$ and $\underline{\underline{\underline{C}}}$ is the fourth-order elasticity tensor. We are looking for the infimum of $\Pi(\underline{u})$

$$
\inf _{\underline{v} \in S} \Pi(\underline{v}), S=\{\underline{v} \in V \mid \gamma(\underline{\underline{\varepsilon}}(\underline{v})) \leq 0\}
$$

The space $S$ is the space of kinematically admissible displacements. The problem (4) is called the primal problem. The introduction of the inequality constraint in the search of the infimum yields to a variational inequality [11].

If $\underline{u}$ is an infimum, then 


$$
\int_{\Omega} \underline{\underline{\varepsilon}}(\underline{u}): \underline{\underline{\underline{C}}}: \underline{\underline{\varepsilon}}(\underline{v}-\underline{u}) d \Omega \geq \int_{\Omega} \underline{f} \cdot(\underline{v}-\underline{u}) d \Omega+\int_{\Gamma_{T}} \underline{t} d \cdot(\underline{v}-\underline{u}) d \Gamma \quad \forall \underline{v} \in S
$$

This result is a variational inequality characterizing the solution $\underline{u}$ of the problem (4). This is also the primal variational formulation of this problem. Unlike classical variational principles encountered in solid mechanics, we obtain an inequality because of the constraint $\underline{u} \in S$.

\section{A classical approach}

\subsection{The variational formulation}

Constrained optimization problems like (4) are difficult to formulate and to solve. Indeed the inequality constraint prevents us from solving it directly.

The goal of this section is to transform a constrained problem into an unconstrained one. In this section, we will recall how our constrained minimization problem can be transformed into saddle point problem using the method of Lagrange multiplier [2] (duality principles). First, to take into account the constraint $\gamma(\underline{\underline{\varepsilon}})$, we add to the elastic strain energy function $\psi(\underline{\underline{\varepsilon}})$ the following characterisic function

$$
\psi(\underline{\underline{\varepsilon}})=\psi^{r}(\underline{\underline{\varepsilon}})+I_{\gamma(\underline{\underline{\varepsilon}})}^{-}
$$

where $\psi^{r}$, is the parent or regular potential $\left(\psi^{r}(\underline{\underline{\varepsilon}})=\frac{1}{2} \underline{\underline{\varepsilon}}: \underline{\underline{\underline{C}}}: \underline{\underline{\varepsilon}}\right)$ and $I^{-}(x)$, is the characteristic function of $\mathbb{R}^{-}$such that

$$
I_{x}^{-}=\left\{\begin{aligned}
0 & \text { if } x \leq 0 \\
+\infty & \text { otherwise }
\end{aligned}\right.
$$

The constitutive law then reads:

$$
\begin{array}{r}
\underline{\underline{\sigma}}=\underline{\underline{\underline{\underline{C}}}: \underline{\underline{\varepsilon}}+p \underline{\underline{I}}} \\
\gamma(\underline{\underline{\varepsilon}}) \leq 0, p \geq 0, \gamma(\underline{\underline{\varepsilon}}) p=0
\end{array}
$$

Now, let the Lagrange multiplier trial functions be $q$. These functions reside in the following space

$$
Q^{+}=\{q \in Q \mid q \geq 0\}, Q=\left\{q \in L^{2}(\Omega)\right\}
$$

Since

$$
I_{\gamma(\underline{\underline{\varepsilon}})}^{-}=\sup _{q \in Q^{+}}\{q \gamma(\underline{\underline{\varepsilon}})\}
$$

the above infimum problem becomes a saddle-point problem with the following functional :

$$
L(\underline{v}, q)=\int_{\Omega}\left[\psi^{r}(\underline{\underline{\varepsilon}})+q \gamma(\underline{\underline{\varepsilon}})\right] d \Omega-\int_{\Omega} \underline{f} \cdot \underline{v} d \Omega-\int_{\Gamma_{t}} \underline{t}_{d} \cdot \underline{v} d \Gamma
$$

We are looking for the saddle-point of this functional 


$$
\inf _{\underline{v} \in V} \sup _{q \in Q^{+}} L(\underline{v}, q)
$$

Unfortunately, there is still an inequality constraint. Indeed, the lagrange multiplier $q$ has to respect the positivity condition $q \geq 0$. We still have to solve a constrained problem.

If $(u, p)$ is the saddle point of the functional $L(\underline{v}, q)$, then

$$
\begin{aligned}
D L(\underline{u}, p)[\underline{v}] & =0 \forall \underline{v} \in V^{0} \\
D L(\underline{u}, p)[q-p] & \leq 0 \forall q \in Q^{+}
\end{aligned}
$$

where $D L(\underline{u}, p)[q-p]$ denotes the directional derivative of $L(u, p)$ with respect to $p$ in the arbitrary direction $(q-p)$ and $D L(\underline{u}, p)[\underline{v}]$ the directional derivative of $L(u, p)$ with respect to the motion in the arbitrary direction $\underline{v}$.

Rewriting (14) and (15), we obtain once again a variational inequality of the form [2]:

$$
\begin{aligned}
\int_{\Omega} \underline{\underline{\varepsilon}}(\underline{u}): \underline{\underline{\underline{C}}: \underline{\underline{\varepsilon}}(\underline{v}) d \Omega+\int_{\Omega} p \operatorname{Tr} \underline{\underline{\varepsilon}}(\underline{v}) d \Omega} & =\int_{\Omega} \underline{f} \cdot \underline{v} d \Omega+\int_{\Gamma_{T}} \underline{t} d \cdot \underline{v} d \Gamma \forall \underline{v} \in V^{0} \\
\int_{\Omega} \gamma(\underline{\underline{\varepsilon}}(\underline{u}))(q-p) d \Omega & \leq 0 \forall q \in Q^{+}
\end{aligned}
$$

An alternative is to use an Augmented Lagrangian formulation [12] which is a composition of an ordinary lagrangian method and a penalty method. This alternative method permits to regularize the saddle point problem [13] and to give an unconstrained problem. Then, we augment once again the functional with a term that ensures the positivity condition [3].

The functional becomes :

$$
\begin{aligned}
L^{+}(\underline{v}, q)= & \int_{\Omega} \psi^{r}(\underline{\underline{\varepsilon}}) d \Omega+\frac{1}{2 \rho} \int_{\Omega}\langle q+\rho \gamma(\underline{\underline{\varepsilon}})\rangle_{+}^{2} d \Omega-\frac{1}{2 \rho} \int_{\Omega} q^{2} d \Omega- \\
& -\int_{\Omega} \underline{f} \cdot \underline{v} d \Omega-\int_{\Gamma_{t}} t_{d} \cdot \underline{v} d \Gamma
\end{aligned}
$$

where $\langle\ldots\rangle_{+}$denotes the positive part of the considered quantity, and $\rho$ is a postive parameter, called augmentation parameter.

The saddle point problem becomes

$$
\inf _{\underline{v} \in V} \sup _{q \in Q} L^{+}(\underline{v}, q)
$$

The constraint on $q$ has been removed and the stationnary condition of the above functional reads:

If $(u, p)$ is the saddle point of the functional $L^{+}(\underline{v}, q)$, then

$$
\begin{aligned}
& D L^{+}(\underline{u}, p)[\underline{v}]=0 \forall \underline{v} \in V^{0} \\
& D L^{+}(\underline{u}, p)[q]=0 \forall q \in Q
\end{aligned}
$$

The stationary condition leads to the following formulation 


$$
\begin{aligned}
\int_{\Omega}[\underline{\underline{\varepsilon}} \underline{\underline{u}}): \underline{\underline{\underline{C}}: \underline{\underline{\varepsilon}}(\underline{v})+\rho \chi(g) \operatorname{Tr} \underline{\underline{\varepsilon}}(\underline{u}) \operatorname{Tr} \underline{\underline{\varepsilon}}(\underline{v})] d \Omega+\int_{\Omega} \chi(g) p \operatorname{Tr} \underline{\underline{\varepsilon}}(\underline{v}) d \Omega=} & \int_{\Omega}[\underline{f} \cdot \underline{v}+\chi(g) \rho \alpha \operatorname{Tr} \underline{\underline{\varepsilon}}(\underline{v})] d \Omega+ \\
& +\int_{\Gamma_{T}} \underline{t} \cdot \underline{v} d \Gamma \forall \underline{v} \in V^{0} \\
\frac{-1}{\rho} \int_{\Omega}(1-\chi(g)) p q d \Omega+\int_{\Omega} \chi(g) \operatorname{Tr} \underline{\underline{\varepsilon}}(\underline{u}) q d \Omega & =\int_{\Omega} \chi(g) \alpha q d \Omega \forall q \in Q
\end{aligned}
$$

where

$$
\chi(x)= \begin{cases}0 & \text { if } x<0 \\ 1 & \text { if } x \geq 0\end{cases}
$$

and $g$ designates the augmented multiplier [3]

$$
g=p+\rho \gamma(\underline{\underline{\varepsilon}})
$$

\begin{tabular}{|c|c|}
\hline \multirow[b]{2}{*}{ mechanical equilibrium } & $\Omega$ \\
\hline & $\underline{\operatorname{div}}(\underline{\underline{\sigma}})+f=\underline{0}$ \\
\hline \multirow{3}{*}{ constitutive relation } & $\underline{\underline{\sigma}} \cdot \underline{n}=\underline{t_{d}}$ \\
\hline & $\underline{\underline{\sigma}}=\underline{\underline{\underline{C}}}: \underline{\underline{\varepsilon}}+\chi(g) g \underline{\underline{I}}$ \\
\hline & $p-\chi(g) g=0$ \\
\hline \multirow{3}{*}{ kinematic conditions } & $g=p+\rho(\operatorname{Tr} \underline{\underline{\varepsilon}}-\alpha)$ \\
\hline & $\underline{u}=\underline{u}_{d}$ \\
\hline & $\underline{\underline{\varepsilon}}=\frac{1}{2}\left(\underline{\underline{\nabla}}(\underline{u})+\underline{\underline{\nabla}}(\underline{u})^{t}\right)$ \\
\hline
\end{tabular}

The parameter $\rho$ does not affect the correctness of the theorical formulation, but from a numerical point of view its choice does affect the convergence of the scheme and the condition number of the tangent stiffness matrix.

The strong form associated to this problem is depicted in Table 1.

Table 1: The strong form

\subsection{Numerical strategy}

\subsubsection{The active-set algorithm}

Starting from the formulation stated previously, we have to solve it numerically. Firstly, this formulation represents a set of non-linear equations even if our setting is the linear elasticity. Indeed, the function $\chi$ presented above introduces a non-linearity. This non-linearity due to the constraint condition could be solved with a Generalized Newton Method [8], but we will use here another method. A kind of fixed point algorithm described in [14, 6, 15, 16, 17, 18] is here used. This algorithm is close to the active set method $[9,10]$ and permits to update iteratively the values of $\chi$. This active-set algorithm is explained below 


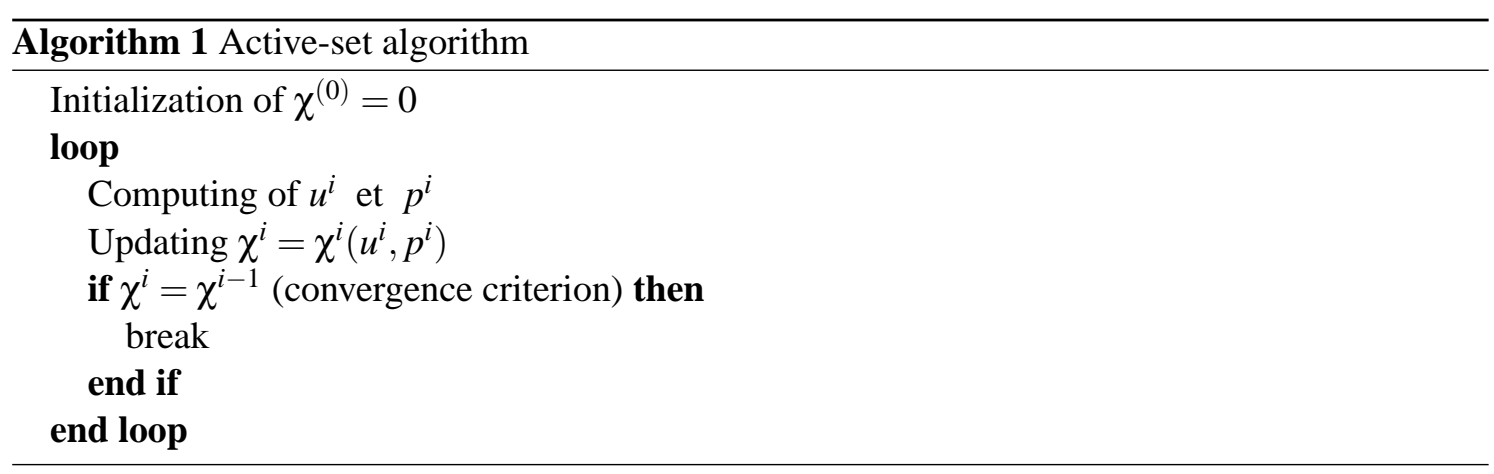

At each step of the loop, the field $\chi$ is completly known over the whole domain. We can then solve a linear problem, and then update the state of $\chi$.

In this algorithm, all the states of the $\chi$ function are changed at the same time. (this function is evaluated at the Gauss points since we use a Gauss quadrature integration). Unfortunaletly, the convergence of this algorithm is proved only if just one state is changed at each iteration. We prefer here to change all the states at the same time [16] so as to obtain a significant gain in speed.

Convergence criterion We evaluate the $\chi$ function at the Gauss points during the numerical integration procedure. The active-set algorithm has converged when the values of $\chi$ at the Gauss points has not changed. Let $\left[\chi^{i}\right]$ the vector containing the values of $\chi$ of all Gauss points at iteration $i$ of the algorithm. The algorithm has converged when $\left[\chi^{i}\right]-\left[\chi^{i-1}\right]=[0]$. Unfortunately, in this algorithm, cases of looping between several states of $\chi$ may occur. These oscillations prevent the algorithm from converging and may deteriorate the accuracy of the results.

\subsubsection{The discretization}

Once the non-linearity due to the presence of the inequality constraint is solved, we have to establish the spatial discretization. We denote $p_{h}$ et $\underline{u}_{h}$ the discrete Lagrange multiplier field and discrete displacement field. The discretized weak form at iteration $i$ of the active set algortithm yields

Find $\left(\underline{u}_{h}, p_{h}\right)$ such that

$$
\begin{gathered}
a\left(\underline{u}_{h}, \underline{v}_{h}\right)+b\left(p_{h}, \underline{v}_{h}\right)=l\left(\underline{v}_{h}\right) \forall \underline{v}_{h} \in V_{h} \\
b\left(\underline{u}_{h}, q_{h}\right)+c\left(p_{h}, q_{h}\right)=p\left(q_{h}\right) \forall q_{h} \in Q_{h}
\end{gathered}
$$

with

$$
\begin{array}{r}
a\left(\underline{u}_{h}^{i}, \underline{v}_{h}\right)=\int_{\Omega_{h}} \underline{\underline{\varepsilon}}\left(\underline{u}_{h}^{i}\right): \underline{\underline{\underline{C}}}: \underline{\underline{\varepsilon}}\left(\underline{v}_{h}\right) d \Omega+\int_{\Omega_{h}} \chi^{i-1} \rho \mathbf{T} \mathbf{T} \underline{\underline{\varepsilon}}\left(\underline{u}_{h}^{i}\right) \operatorname{Tr} \underline{\underline{\varepsilon}}\left(\underline{v}_{h}\right) d \Omega \\
\left.b\left(p_{h}^{i}, \underline{v}_{h}\right)=\int_{\Omega_{h}} \chi^{i-1} p_{h}^{i} \operatorname{Tr} \underline{\underline{\varepsilon}} \underline{\underline{v}} \underline{v}_{h}\right) d \Omega \\
l\left(\underline{v}_{h}\right)=\int_{\Omega_{h}} \underline{f} \cdot \underline{v}_{h} d \Omega+\int_{\Gamma_{T}} \underline{t}_{d} \cdot \underline{v}_{h} d \Gamma+\int_{\Omega_{h}} \chi^{i-1} \rho \alpha \operatorname{Tr} \underline{\underline{\varepsilon}}\left(\underline{v}_{h}\right) d \Omega \\
c\left(p_{h}^{i}, q_{h}\right)=\frac{-1}{\rho} \int_{\Omega_{h}}\left(1-\chi^{i-1}\right) p_{h}^{i} q_{h} d \Omega \\
b\left(\underline{\underline{u}}_{h}^{i}, q_{h}\right)=\int_{\Omega_{h}} \chi^{i-1} \operatorname{Tr} \underline{\underline{\varepsilon}}\left(\underline{u}_{h}^{i}\right) q_{h} d \Omega \\
p\left(q_{h}\right)=\int_{\Omega_{h}} \chi^{i-1} \alpha q_{h} d \Omega
\end{array}
$$

where $Q_{h}$ and $V_{h}$ are the discrete finite dimensions subspaces of $Q$ and $V$. 
The discretization of the weak form involves two fields : the displacement $\underline{u}$ and the Lagrange multiplier $p$. The shape functions for the Lagrange multiplier differ from those for the displacements. Hence, we will use different symbols for the two approximations. The classical FEM discretization on an element $\Omega_{e}$ reads

$$
\begin{aligned}
\left.\underline{u}^{h}\right|_{\Omega_{e}} & =\sum_{i=1}^{N} \underline{u}_{i}^{h} N_{i}^{u} \\
\left.p^{h}\right|_{\Omega_{e}} & =\sum_{i=1}^{N^{\prime}} p_{i}^{h} N_{i}^{p}
\end{aligned}
$$

where $N$ and $N^{\prime}$ is the number of coefficients describing the approximation, and $N_{i}$ is the shape function associated to the coefficient $i$. If the use of a Lagrange multiplier field ensures the respect of the constraint, its discretization involves a major problem. Indeed, the interpolation used has to respect the well-known Ladyzhenskaya-Babuska-Brezzi compatibility condition [19] which guarantees the stability of the mixed method.

Denoting

$$
b^{\prime}\left(\underline{v}_{h}, q_{h}\right)=\int_{\Omega} \operatorname{Tr} \underline{\underline{\varepsilon}}\left(\underline{v}_{h}\right) q_{h} d \Omega
$$

This condition reads

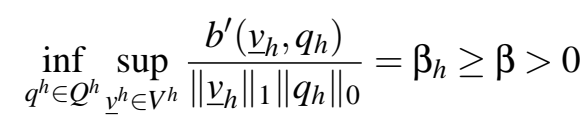

Where $\|.\|_{1}$ and $\|.\|_{0}$ designate respectivlely the $H^{1}$ and $L^{2}(\Omega)$ norms, and $\beta$ is a positive real independent of the mesh size.

Among all mixed elements, only a few respect this condition. Moreover, we have used mixed elements with discontinuous pressure because we have noticed that this kind of elements permits the stability of the active-set algorithm. These elements can be used because we do not need the derivative of the pressure field in the weak formulation. One basic discontinuous pressure element has been selected. The mixed element chosen is the $P 2^{+} / P 1_{\text {disc }}$ (see Figure 2). This element is composed of a quadratic displacement enriched by a cubic bubble function and a linear discontinuous pressure. The upper script + designates the enrichment by the bubble function. The displacement is enriched with this special function so as to increase the degree of the displacement interpolation and to ensure the stability of the formulation.

More preciselly, the shape functions for each component of the displacement field are the regular second order lagrange shape function (P2), plus a bubble function. The bubble function that we used is defined in each triangle as : $b(\underline{x})=\lambda_{1}(\underline{x}) \lambda_{2}(\underline{x}) \lambda_{3}(\underline{x})$ where the $\lambda_{i}$ are the barycentric coordinates relative to the three nodes of the triangle. The pressure field is defined independently in each element by a linear combination of the three order one lagrange shape function on the triangle. This finite element formulation therefore give a total number of degree of freedom $n d o f=2 * n v+$ $2 * n e+(2+3) * n t$, where $n v$ is the number of vertices, $n e$ is the number of edges and $n t$ is the number of triangle.

\section{A new approach}

\subsection{The strategy}

The essential difficulty of the problem described in section 2 is that the region where the constraint is active is not known a priori. This observation has motivated a new strategy of resolution. 


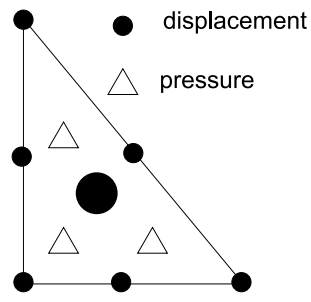

Figure 2: $P 2^{+} / P 1_{\text {disc }}$ mixed element

We consider here the same domain $\Omega$ and the same boundary conditions as before. But the strategy proposed here is to split the whole domain $\Omega$ into two distinct subdomains separated by $\Gamma$. The first domain denoted by $\Omega_{c}$ is the constrained zone or active zone where an equality constraint is imposed. The second domain denoted by $\Omega_{f}$ is the unconstrained or unactive zone, where no constraints are imposed.

$$
\begin{aligned}
\Omega_{c} \cup \Omega_{f} & =\Omega \\
\Omega_{c} \cap \Omega_{f} & =\emptyset \\
\partial \Omega_{c} \cap \partial \Omega_{f} & =\Gamma
\end{aligned}
$$

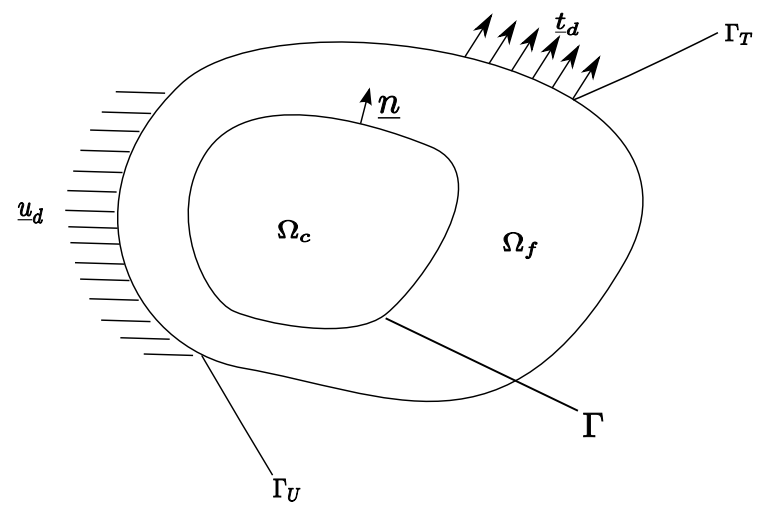

Figure 3: Problem statement 2

On each subdomain, the same linear elastic isotropic homogeneous material is considered. But in the domain $\Omega_{c}$ we impose the following constraint

$$
\gamma(\underline{\underline{\varepsilon}})=\operatorname{Tr} \underline{\underline{\varepsilon}}-\alpha=0 \text { on } \Omega_{c}
$$

The elastic energy in domains $\Omega_{f}$ and $\Omega_{c}$ are given by

$$
\begin{aligned}
& \psi_{c}=\frac{1}{2} \underline{\underline{\varepsilon}}: \underline{\underline{\underline{C}}}: \underline{\underline{\varepsilon}}+I_{\gamma(\underline{\underline{\varepsilon}})}^{0}=\mu \underline{\underline{\varepsilon}}^{D}: \underline{\underline{\varepsilon}}^{D}+\frac{\kappa}{2} \alpha^{2}+I_{\gamma(\underline{\underline{\varepsilon}})}^{0} \\
& \psi_{f}=\frac{1}{2} \underline{\underline{\varepsilon}}: \underline{\underline{\underline{C}}}: \underline{\underline{\varepsilon}}=\mu \underline{\underline{\varepsilon}}^{D}: \underline{\underline{\varepsilon}}^{D}+\frac{\kappa}{2}(\operatorname{Tr} \underline{\underline{\varepsilon}})^{2}
\end{aligned}
$$

where $\kappa=\lambda+\frac{2}{3} \mu$ is the bulk modulus, with $\lambda$ and $\mu$ the Lamé coefficients and $I^{0}(x)$, is the characteristic function of 0 such that

$$
I^{0}(x)=\left\{\begin{aligned}
0 & \text { if } x=0 \\
+\infty & \text { otherwise }
\end{aligned}\right.
$$


The corresponding constitutive laws then read

$$
\begin{array}{r}
\underline{\underline{\sigma}}_{c}=2 \mu \underline{\underline{\varepsilon}}^{D}+\kappa \alpha \underline{\underline{I}}+p \underline{\underline{I}} \\
\underline{\underline{\sigma}}_{l}=2 \mu \underline{\underline{\varepsilon}}^{D}+\kappa \operatorname{Tr} \underline{\underline{\varepsilon}}
\end{array}
$$

Note that $p$ is not the classical pressure (Tr$\underline{\sigma}$ ), it is what is needed for $\underline{\sigma}$ to be in equilibrium We can notice that the behaviour in domain $\Omega_{f}$ and $\Omega_{c}$ no longer involve inequalities and differ from the original behaviour (8).

The total potential energy $\Pi^{\prime}(\underline{u})$ of the system reads

$$
\Pi^{\prime}(\underline{u})=\int_{\Omega_{c}} \psi_{c}(\underline{\underline{\varepsilon}}) d \Omega+\int_{\Omega_{f}} \psi_{f}(\underline{\underline{\varepsilon}}) d \Omega-\int_{\Omega} \underline{f} \cdot \underline{u} d \Omega-\int_{\Gamma_{T}} \underline{t}_{d} \cdot \underline{u} d \Gamma
$$

The interface between the two domains can be seen as a surface of discontinuity and the strategy is to propagate correctly the front separating the two elastic phases $\Omega_{c}$ and $\Omega_{f}$ so as to find the exact location of $\Gamma$. Then, we have replaced the initial problem of a media subjected to a kinematic inequality constraint by a problem of a media subjected to an equality constraint over an unknown restricted domain $\Omega_{c}$.

The interface $\Gamma$ is a coherent interface, thus the jump of the displacement field $\underline{u}$ has to vanish across the interface $\Gamma$, preventing separation, penetration or slipping of the two domains. We do have the continuity of the normal stress vector.

Let $\Gamma$ be fixed for the moment. We are looking for the infimum of $\Pi^{\prime}(\underline{u})$

$$
\inf _{\underline{v} \in V} \Pi^{\prime}(\underline{v})
$$

Rewriting (47) as the following saddle point problem

$$
\inf _{\underline{v} \in V} \sup _{q \in Q} L^{\prime}(v, q)
$$

where $L^{\prime}(v, q)$ designates the following Lagrangian functional

$$
L^{\prime}(v, q)=\int_{\Omega_{c}}\left[\psi_{c}(\underline{\underline{\varepsilon}})+q \gamma(\underline{\varepsilon})\right] d \Omega+\int_{\Omega_{f}} \psi_{f}(\underline{\underline{\varepsilon}}) d \Omega-\int_{\Omega} \underline{f} \cdot \underline{u} d \Omega-\int_{\Gamma_{T}} \underline{t} d \underline{u} d \Gamma
$$

The stationary condition of the above functional leads to the variational form

Find $(\underline{u}, p)$ such that:

$$
\begin{aligned}
& a(\underline{u}, \underline{v})+ b(p, \underline{v})=l(\underline{v}) \forall \underline{v} \in V^{0} \\
& b(\underline{u}, q)=p(q) \forall q \in Q \\
& a(\underline{u}, \underline{v})=\int_{\Omega} \underline{\underline{\varepsilon}}(\underline{u}): \underline{\underline{\underline{C}}}: \underline{\underline{\varepsilon}}(\underline{v}) d \Omega \\
& b(p, \underline{v})=\int_{\Omega_{c}} p \operatorname{Tr} \underline{\underline{\varepsilon}}(\underline{v}) d \Omega \\
& l(\underline{u}, \underline{v})=\int_{\Omega} \underline{f} \cdot \underline{v} d \Omega+\int_{\Gamma_{T}} \underline{\underline{t}} d \cdot \underline{v} d \Gamma \\
& b(\underline{u}, q)=\int_{\Omega_{c}} \operatorname{Tr} \underline{\underline{\varepsilon}}(\underline{u}) q d \Omega \\
& p(q)=\int_{\Omega_{c}} \alpha q d \Omega
\end{aligned}
$$


The strong formulation associated to this new problem reads:

\begin{tabular}{|c|c|c|c|}
\hline & $\Omega_{c}$ & $\Gamma$ & $\Omega_{f}$ \\
\hline mechanical equilibrium & $\begin{array}{c}\underline{\operatorname{div}}(\underline{\underline{\sigma}})+\underline{f}=\underline{0} \\
\underline{\underline{\sigma}} \cdot \underline{n}=\underline{t}_{d} \text { on } \partial \Omega_{c} \cap \Gamma_{T}\end{array}$ & {$[[\underline{\underline{\sigma}} \cdot \underline{n}]]=\underline{0}$} & $\begin{array}{c}\underline{\operatorname{div}}(\underline{\underline{\sigma}})+\underline{f}=\underline{0} \\
\underline{\underline{\sigma}} \cdot \underline{n}=\underline{t}_{d} \text { on } \partial \Omega_{f} \cap \Gamma_{T}\end{array}$ \\
\hline constitutive relation & $\begin{array}{c}\underline{\underline{\sigma}}=\underline{\underline{\underline{\underline{C}}}}: \underline{\underline{\varepsilon}}+p \underline{\underline{I}} \\
\operatorname{Tr} \underline{\underline{\varepsilon}}-\alpha=0\end{array}$ & & $\underline{\underline{\sigma}}=\underline{\underline{\underline{C}}}: \underline{\underline{\varepsilon}}$ \\
\hline kinematic conditions & $\begin{array}{l}\underline{u}=\underline{u}_{d} \text { on } \partial \Omega_{c} \cap \Gamma_{U} \\
\underline{\underline{\varepsilon}}=\frac{1}{2}\left(\underline{\underline{\nabla}}(\underline{u})+\underline{\underline{\nabla}}(\underline{u})^{t}\right)\end{array}$ & $\begin{array}{c}{[[\underline{u}]]=\underline{0}} \\
\underline{\underline{\varepsilon}}=\frac{1}{2}\left(\underline{\underline{\nabla}}(\underline{u})+\underline{\underline{\nabla}}(\underline{u})^{t}\right)\end{array}$ & $\begin{array}{l}\underline{u}=\underline{u}_{d} \text { on } \partial \Omega_{f} \cap \Gamma_{U} \\
\underline{\underline{\varepsilon}}=\frac{1}{2}\left(\underline{\underline{\nabla}}(\underline{u})+\underline{\underline{\nabla}}(\underline{u})^{t}\right)\end{array}$ \\
\hline
\end{tabular}

Table 2: The $\Gamma$ problem

Unlike the previous approach, we obtain a fully linear set of equations. Indeed, the nonlinearity introduced by the function $\chi$ has been removed. The intrinsec non-linearity due to the presence of the inequality constraint will be solved by changing iteratively the shape of each phase. Note that this formulation does no involve any parameter since the equality constraint on $\Omega_{c}$ is solved using an ordinary Lagrangian method.

We denote

$$
\psi(\varepsilon(\underline{x}), \underline{x})=\left\{\begin{array}{cc}
\psi_{c}\left(\varepsilon_{c}(\underline{x})\right) & \text { if } \underline{x} \in \Omega_{c} \\
\psi_{f}\left(\varepsilon_{f}(\underline{x})\right) & \text { if } \underline{x} \in \Omega_{f}
\end{array}\right.
$$

Let us consider now the directional derivative of $\Pi^{\prime}(\underline{u})$ for a configurational change $\underline{\theta}$ of $\Gamma$. Here, $\underline{u}$ is the solution of the infimum problem (47) for a given location $\Gamma$ of the interface. We could then write $\Pi^{\prime}(\underline{u})=\Pi^{\prime}(\underline{u}(\Gamma), \Gamma)$.

Following [20], we get:

$$
\begin{aligned}
D \Pi^{\prime}(\underline{u}(\Gamma), \Gamma)[\underline{\theta}]= & \int_{\Omega_{f}} D \psi_{f}[\underline{\theta}] d \Omega+\int_{\Omega_{c}} D \psi_{c}[\underline{\theta}] d \Omega+\int_{\Omega_{f}} \psi_{f} D(d \Omega)[\underline{\theta}]+\int_{\Omega_{c}} \psi_{c} D(d \Omega)[\underline{\theta}]- \\
& -\int_{\Gamma_{t}} t_{d} \cdot D \underline{u}[\underline{\theta}] d \Gamma-\int_{\Omega} D \underline{f}[\underline{\theta}] \cdot \underline{u} d \Omega-\int_{\Omega} \underline{f} \cdot D \underline{u}[\underline{\theta}] d \Omega-\int_{\Omega} \underline{f} \cdot \underline{u} D(d \Omega)[\underline{\theta}](57) \\
D \Pi^{\prime}(\underline{u}(\Gamma), \Gamma)[\underline{\theta}]= & \int_{\Omega_{f}} \underline{\underline{\sigma}}: \underline{\underline{\varepsilon}}(D \underline{u}[\underline{\theta}]) d \Omega+\int_{\Omega_{c}} \underline{\underline{\sigma}}: \underline{\underline{\varepsilon}}(D \underline{u}[\underline{\theta}]) d \Omega-\int_{\Gamma_{t}} \underline{t} \cdot D \underline{u}[\underline{\theta}] d \Gamma-\int_{\Omega} \underline{f} \cdot D \underline{u}[\underline{\theta}] d \Omega+ \\
& +\int_{\Omega_{f}}\left(\psi_{f}-\underline{f} \cdot \underline{u}\right) \nabla \cdot \underline{\theta}-\underline{\underline{\sigma}}:(\nabla \underline{u} \cdot \nabla \underline{\theta})-D \underline{f}[\underline{\theta}] \cdot \underline{u} d \Omega+ \\
& +\int_{\Omega_{c}}\left(\psi_{c}-\underline{f} \cdot \underline{u}\right) \nabla \cdot \underline{\theta}-\underline{\underline{\sigma}}:(\nabla \underline{u} \cdot \nabla \underline{\theta})-D \underline{f}[\underline{\theta}] \cdot \underline{u} d \Omega
\end{aligned}
$$

The first four term above in the right hand side cancel out because of the equilibrium. Then noting the expression of the Eshelby tensor [21] $\underline{\underline{P}}$,

$$
\underline{\underline{P}}=(\psi(\underline{\underline{\varepsilon}})-\underline{f} \cdot \underline{u}) \underline{\underline{I}}-\underline{\underline{\nabla}} \underline{u}^{T} \cdot \underline{\underline{\sigma}}
$$


we get

$$
D \Pi^{\prime}(\underline{u}(\Gamma), \Gamma)[\underline{\theta}]=\int_{\Gamma} \underline{\theta} \cdot[[\underline{\underline{P}}]] \cdot \underline{n} d \Gamma
$$

After calculus (Appendix A), we get

$$
D \Pi^{\prime}(\underline{u}(\Gamma), \Gamma)[\underline{\theta}]=\int_{\Gamma} \tau \theta d \Gamma
$$

where

$$
\tau=(\lambda+2 \mu)^{-1} \frac{p_{c}^{2}}{2}=\frac{(\lambda+2 \mu)}{2}\left(\operatorname{Tr} \underline{\underline{\varepsilon}}_{f}-\alpha\right)^{2}
$$

Remark The term $\tau$ is the driving force or the configurational force acting on the interface $\Gamma$. One can notice that the configurational force is always positive whatever the location of the

\begin{tabular}{|c|c|c|c|}
\hline & $\Omega_{c}$ & $\Gamma$ & $\Omega_{f}$ \\
\hline configurational equilibrium & $\underline{\operatorname{div}} \underline{\underline{P}}+\underline{\underline{\nabla}} \underline{f} \cdot \underline{u}=0$ & $\tau=0$ & $\underline{\operatorname{div}} \underline{\underline{P}}+\underline{\underline{\nabla}} \underline{f} \cdot \underline{u}=0$ \\
\hline optimality conditions & $p_{c} \geq 0$ & & $\operatorname{Tr} \underline{\underline{\varepsilon}}_{f}-\alpha \leq 0$ \\
\hline
\end{tabular}
interface.

The exact location of the interface is the one that verify the following optimality criteria.

Table 3 : The $\Gamma$ optimality

Indeed, if the configurational equilibrium is satsified, then $p$ is continous accross $\Gamma$ and $p \geq 0$. Moreover, $\operatorname{Tr} \underline{\underline{\varepsilon}}$ is also continous accross $\Gamma$ and $\operatorname{Tr} \underline{\underline{\varepsilon}} \leq \alpha$. In this case, the couple $(\underline{u}, p)$ verify the equilibrium conditions, the boundary conditions, and the Karush-Kuhn-Tucker conditions of optimality (9). Hence, $(\underline{u}, p)$ is the solution of the problem (4). The final algorithm, therefore will consist in finding the position of $\Gamma$ such as the configurational equilibrium defined in Table 3 is ensured. Then, we verify the conditions of optimality exposed in Table 3. Around the points where these two conditions are violated, a new trial boundary needs to be introduced. We then evolve once again the whole new interface so as to verify the configurational equilibrium.

\subsection{Analytical solution on a one-dimensional example}

Let us consider the simple one dimensional problem in the small strain elastostatic setting with uniaxial state of strain as shown in Figure 4. We wish to limit the uniaxial strain of a truss by a value $\alpha$ under a linear prescribed load $f(x)=f x$.

We split the bar into two domains. The constrained domain $[0, y]$ and the unconstrained domain $[y, L]$.

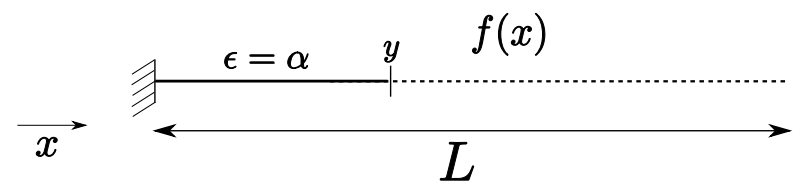

Figure 4: A one-dimensional example 
The exact displacement, strain, and pressure field expression along the bar for any given location $y$ of the interface are given in Appendix B.

We choose $\alpha$ such that the exact location of the interface $y_{e}$ is at the middle of the bar.

$$
\alpha=\frac{3 f}{8(\lambda+2 \mu)} L^{2}
$$

Denoting $\tilde{\varepsilon}=\frac{\varepsilon(\lambda+2 \mu)}{f L^{2}}$ and $\tilde{\sigma}=\frac{\sigma}{f L^{2}}$, the exact strain and stress fields $\tilde{\varepsilon}$ and $\tilde{\sigma}$ are depicted in Figure 6.

The evolution of the potential energy and the configurational force with the location of the interface are depicted in Figure 5 and the analytical expression are given in Appendix B.
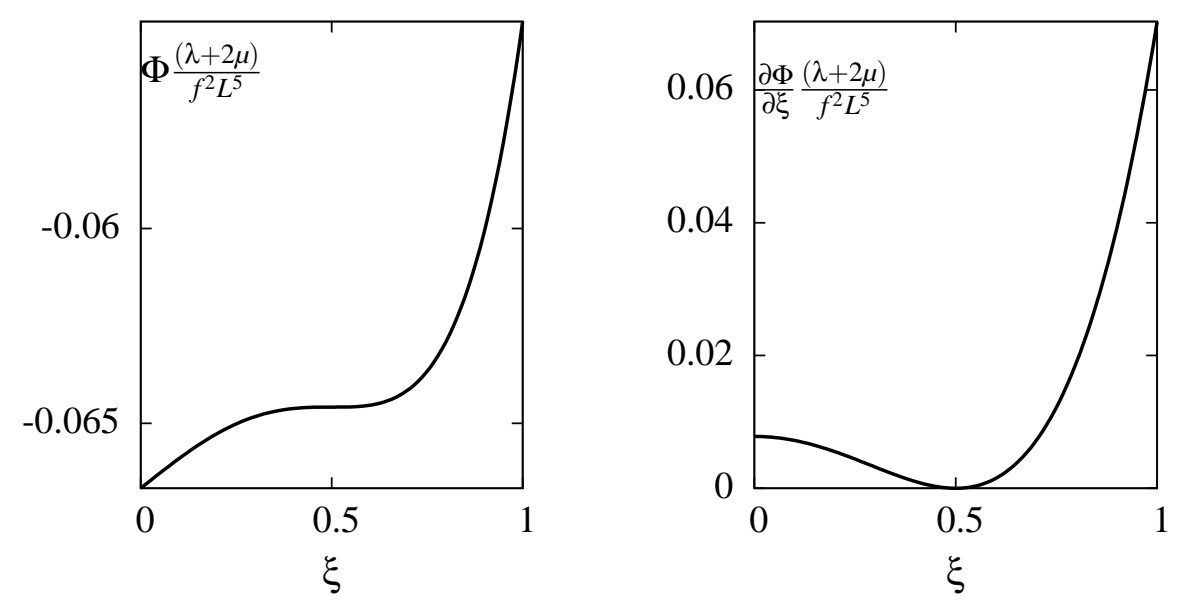

Figure 5: Evolution of the potentiel energy (left) and its derivative (right) with $\xi$
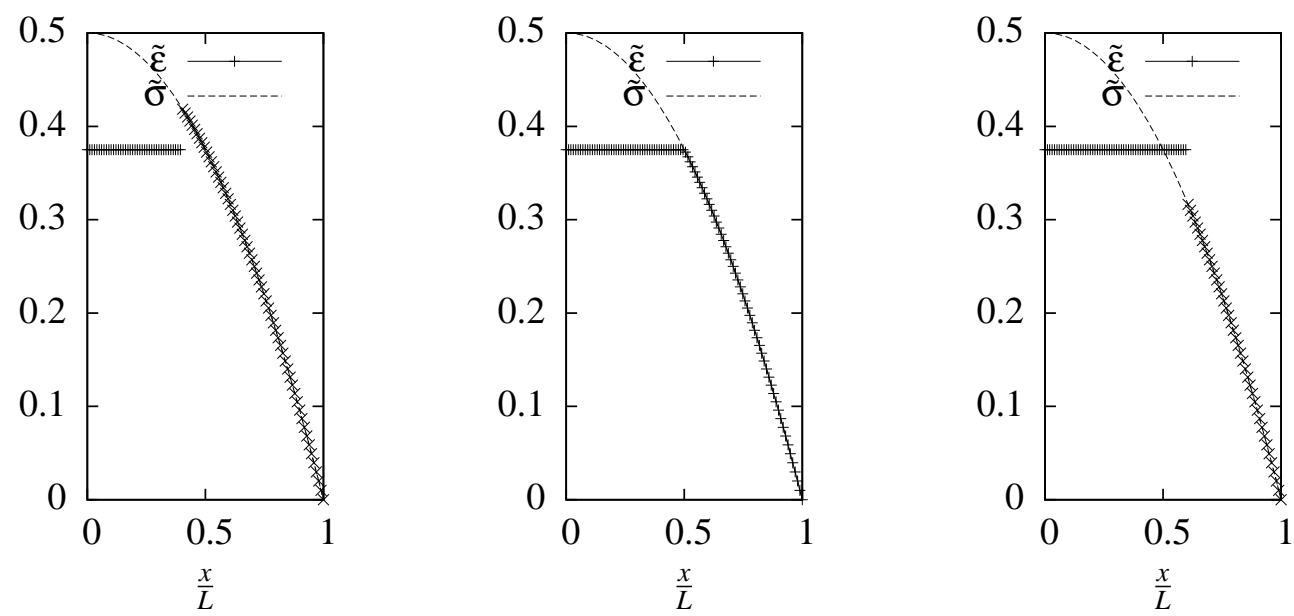

Figure 6: The strain and stress field for $\xi<\frac{1}{2}$ (left), $\xi=\frac{1}{2}$ (middle), $\xi>\frac{1}{2}$ (right)

When the interface is not located at the exact position, the strain field is discontinuous at the interface. On can notice that the potential energy of the system is stationary, when the interface is located at $\xi=\frac{1}{2}$. It means that at the exact location of the interface, the configurational force is zero, as one can see in Figure 5. Finally, one can see that the configurational force is always positive whatever the location of the interface and is minimum at the exact location. Hence, the total potential energy is always increasing with the location of the interface $\xi$. The exact location makes the potential energy stationary but not minimum as one can see in Figure 5. 


\subsection{Shape sensitivity}

The goal of this section is to establish the change in $(\underline{u}, p)$ as a result of the migration of the interface. Consider the perturbed constrained zone $\Omega_{c}^{\tau}$ and the perturbed unconstrained zone $\Omega_{f}^{\tau}$. Let us introduce a velocity field $\underline{\theta}$ responsible for the migration of the interface and the following transformation:

$$
\begin{array}{r}
\Omega_{c} \rightarrow \Omega_{c}^{\tau} \\
\underline{x}_{\tau}=\underline{x}+\tau \underline{\theta}(\underline{x}) \forall \underline{x} \in \Omega_{c} \\
\Omega_{f} \rightarrow \Omega_{f}^{\tau} \\
\underline{x}_{\tau}=\underline{x}+\tau \underline{\theta}(\underline{x}) \forall \underline{x} \in \Omega_{f}
\end{array}
$$

The shape change of each phase is expressed using a sufficiently smooth velocity field $\underline{\theta}$ and a parameter $\tau$. The trick introduced in [25] is to use the classical continuum terminology and assimilate the change of shape to a motion of a body. We rewrite the state equation (50) with the parameter $\tau$.

$$
\begin{aligned}
a(\tau, \underline{u}, \underline{v})+b(\tau, p, \underline{v}) & =l(\tau, \underline{v}) \forall \underline{v} \in V^{0} \\
b(\tau, \underline{u}, q) & =p(\tau, q) \forall q \in Q
\end{aligned}
$$

Taking the derivative of the above equation with respect to $\tau$

$$
\begin{array}{r}
a\left(\tau, \frac{\partial \underline{u}}{\partial \tau}, \underline{v}\right)+b\left(\tau, \frac{\partial p}{\partial \tau}, \underline{v}\right)=\frac{\partial l(\tau, \underline{v})}{\partial \tau}-\frac{\partial a(\tau, \underline{u}, \underline{v})}{\partial \tau}-\frac{\partial b(\tau, \underline{u}, q)}{\partial \tau} \forall \underline{v} \in V^{0} \\
b\left(\tau, \frac{\partial \underline{u}}{\partial \tau}, q\right)=\frac{\partial p(\tau, q)}{\partial \tau}-\frac{\partial b(\tau, \underline{u}, q)}{\partial \tau} \forall q \in Q
\end{array}
$$

Defining $\frac{\partial u}{\partial \tau}=\underline{\dot{u}}$ and $\frac{\partial p}{\partial \tau}=\dot{p}$, the above formulation becomes

Find $(\underline{\dot{u}}, \dot{p})$ such that:

$$
\begin{aligned}
& \int_{\Omega} \underline{\underline{\varepsilon}}(\underline{\dot{u}}): \underline{\underline{\underline{C}}}: \varepsilon(\underline{v}) d \Omega+\int_{\Omega_{c}} \dot{p} \operatorname{Tr} \varepsilon(\underline{v}) d \Omega=\int_{\Omega_{c}} p \nabla \underline{\theta}^{T}: \nabla \underline{v} d \Omega-\int_{\Omega_{c}} p \operatorname{div}(\underline{\theta}) \operatorname{Tr}(\nabla \underline{v}) d \Omega+ \\
& +\int_{\Gamma_{T}} \underline{t}_{d}\left(-\underline{n} \cdot \underline{\theta}^{T} \underline{n}^{T}+\operatorname{div}(\underline{\theta})\right) \underline{v} d \Gamma+ \\
& +\int_{\Omega} f(\underline{x}) \operatorname{div}(\underline{\theta}) \cdot \underline{v} d \Omega+\int_{\Omega}(\nabla \underline{f} \underline{\theta}) \cdot \underline{v} d \Omega+ \\
& +\int_{\Omega} \underline{T}: \nabla \underline{v} d \Omega \forall \underline{v} \in V^{0}
\end{aligned}
$$

with $\underline{\underline{T}}=\left[\underline{\underline{\underline{C}}}:(\nabla \underline{u} \nabla \underline{\theta})_{s}+\underline{\underline{\underline{C}}}: \nabla_{s} \underline{u} \nabla \underline{\theta}^{T}-\underline{\underline{\underline{C}}}: \nabla_{s} \underline{u} \operatorname{div}(\underline{\theta})\right]$

$$
\begin{aligned}
\int_{\Omega_{c}} \operatorname{Tr} \varepsilon(\underline{\dot{u}}) q d \Omega= & \int_{\Omega_{c}} \operatorname{Tr}\left((\nabla \underline{u} \nabla \underline{\theta})_{s}\right) q d \Omega-\int_{\Omega_{c}} \operatorname{Tr}\left(\nabla_{s} \underline{u}\right) d i v(\underline{\theta}) q d \Omega+ \\
& +\int_{\Omega_{c}} \alpha \operatorname{div}(\underline{\theta}) q d \Omega \forall q \in Q
\end{aligned}
$$

We can notice that the two bilinear forms of (66) are similar to the bilinear forms of (50). Only the linear forms on the right hand side are different and they imply the knowledge of the fields $(\underline{u}, p)$ and the velocity field $\underline{\theta}$. This weak form can be discretized as the previous one.

If we use the same approximation for the two couple of fields $(\underline{\dot{u}}, \dot{p})$ and $(\underline{u}, p)$, we will obtain two linear systems to solve with the same tangent stiffness matrix. From a numerical point of view, this remark has a huge importance, since we will not have to compute twice the tangent matrix. If we use a LU solver for the two linear systems, we then make the LU decomposition just once. 


\subsection{Numerical Strategy}

The goal of this section is to present the numerical tools and the numercial procedure to move the interface $\Gamma$ towards its exact location.

\subsubsection{Level-set/X-FEM}

To represent the surface of discontinuity separating the domain $\Omega_{c}$ et $\Omega_{f}$ we used the notion of level-set $[23,22]$. The level-set method permits an implicit representation of surfaces through a higher dimensional function $\phi(\underline{x})$.

A level-set is a function that evaluates at each point of the domain the signed distance to the surface of discontinuity. The surface $\Gamma$ is then defined by

$$
\Gamma=\{\underline{x} \mid \phi(\underline{x})=0\}
$$

By convention, the sign of the level-set is negative if the point is located in $\Omega_{c}$ and positive if the point is located in $\Omega_{f}$.

$$
\begin{aligned}
& \phi(\underline{x})<0 \text { if } \underline{x} \in \Omega_{c} \\
& \phi(\underline{x})>0 \text { if } \underline{x} \in \Omega_{f}
\end{aligned}
$$

The iso-zero of the level-set locates the true location of the interface. The level-set is evaluated at the nodes of the mesh and we interpolate these values with the classical linear interpolation functions $N_{i}$.

$$
\phi(\underline{x})=\sum_{i} N_{i}(\underline{x}) \phi_{i}
$$

Because of the presence of the surface of discontinuity $\Gamma$, the strain tensor field presents a discontinuity. To avoid poor rates of convergence we enrich the classical approximation through a partition of unity technique for strain jump [5]. The classical discretization (34) becomes

$$
\left.\underline{u}^{h}\right|_{\Omega_{e}}=\sum_{i=1}^{N} \underline{u}_{i}^{h} N_{i}^{u}+\sum_{j=1}^{N^{\prime}} N_{j}^{u} F(\underline{x}) b_{j}
$$

Additional degrees of freedom $b_{j}$ have been added at the nodes for which the support is split by the interface. The enrichment function $F$, is chosen such that its gradient is discontinuous along $\Gamma$. Thus, we can represent the discontinuity in strain. The function $F$ is defined as [5]:

$$
F(\underline{x})=\sum_{i}\left|\phi_{i}\right| N_{i}(\underline{x})-\left|\sum_{i} \phi_{i} N_{i}(\underline{x})\right|
$$

The function $F$ is depicted in Figure 7, for a one dimensional case.

In our case, we enrich the linear part of the approximation. It means that the $\mathbf{N}_{j}^{u}$ functions are the classical linear interpolation function even if the $\mathbf{N}_{i}^{u}$ are quadratic. The pressure field $p$ is only defined over the domain $\Omega_{c}$. To take into account the presence of $\Gamma$, the weak form involving $p$ is integrated only on $\Omega_{c}$. Thanks to the level-set notion, the interface $\Gamma$ do not need to be meshed. We just have to take care of the integration step as desribed in [4]. 


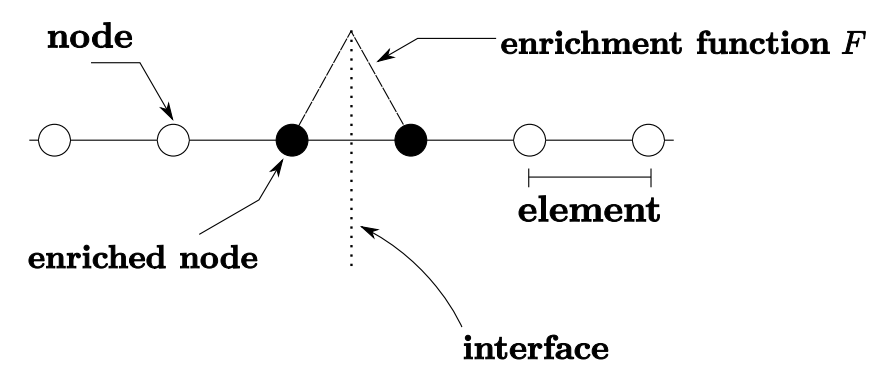

Figure 7: The enrichment function

\subsubsection{Front evolution}

We have shown in section 4.1 that the condition in Table 3 ensures that $(\underline{u}, p)$ is the solution of (4). Numerically, we will first find the location of the interface that ensures the condition $\tau=0$. When this location is found, we verify the two other one.

Thanks to (62), the condition $\tau=0$ on $\Gamma$ is equivalent to the condition $p=0$ on $\Gamma$. Rewriting this condition as a variational form, we get

$$
\int_{\Gamma} p q^{*} d \Gamma=0 \forall q^{*} \in L^{2}(\Gamma)
$$

We now take the directional derivative of (75) for a configurational change $\underline{v}$, where $\underline{v}$ is a normal velocity responsible for the migration of the interface $\Gamma$.

$$
D\left(\int_{\Gamma} p q^{*} d \Gamma\right)[\underline{v}]=\int_{\Gamma} D p[\underline{v}] q^{*}+\int_{\Gamma} p q^{*} D d \Gamma[\underline{v}]
$$

Since

$$
\operatorname{Dd} \Gamma[\underline{v}]=\nabla_{s u r} \cdot \underline{v}=\vartheta \underline{v} \cdot \underline{n}
$$

where $\vartheta$ denotes the curvature of the interface and $\nabla_{\text {sur }} \underline{\underline{v}}$ is the surfacic divergence of $\underline{v}$

We thus get

$$
D\left(\int_{\Gamma} p q^{*} d \Gamma\right)[\underline{v}]=\int_{\Gamma} D p[\underline{v}] q^{*} d \Gamma+\int_{\Gamma} \vartheta p \underline{v} \cdot \underline{n} q^{*} d \Gamma
$$

The algorithmic scheme is the following. We first initialize $\Gamma=\Gamma_{0}$. And then at iteration $k$, we calculate the velocity $\underline{v}_{k}$ using the following Newton-Raphson procedure

$$
\begin{array}{r}
\int_{\Gamma_{k}} p q^{*} d \Gamma+D\left(\int_{\Gamma_{k}} p q^{*} d \Gamma\right)\left[\underline{v}_{k}\right]=0 \\
\int_{\Gamma_{k}} p q^{*} d \Gamma+\int_{\Gamma_{k}} D p\left[\underline{v}_{k}\right] q^{*} d \Gamma+\int_{\Gamma_{k}} \vartheta p \underline{v}_{k} \cdot \underline{n} q^{*} d \Gamma=0
\end{array}
$$

If we use a modal representation for the velocity field $\underline{v}_{k}$ and the test functions $q^{*}$

$$
\begin{aligned}
\underline{v}_{k} & =\sum_{i} F_{i} \tilde{v}_{i} \underline{n} \\
q^{*} & =\sum_{i} F_{i} \tilde{q}_{i}^{*}
\end{aligned}
$$


The modes $F_{i}$ are functions defined over the interface $\Gamma$. To do so, $\Gamma$ is parametrized using a curvilinear abscissa $s \in[0,2 \pi]$. Two types of modes are here introduced to describe the field.

- cosinus modes

$$
F_{i}=\cos (i s)
$$

- sinus modes

$$
F_{i}=\sin (i s)
$$

If we neglect the curvature term $\int_{\Gamma_{k}} \vartheta p \underline{v}_{k} \cdot \underline{n} d \Gamma$ that becomes small as $p$ tends to zero, we get

$$
\int_{\Gamma_{k}} D p\left[\underline{v}_{k}\right] q^{*} d \Gamma=-\int_{\Gamma_{k}} p q^{*} d \Gamma
$$

that leads to the following matricial system

$$
[S][\tilde{v}]=[P]
$$

where $[S]$ is the matrix containing the sensitivity of $p$ to the migration of the interface for each modes $F_{i} \underline{n}$, and $[P]$ is the vector containing the coefficients of the modal projection of the pressure field $p$ on the interface $\Gamma$.

We now present the procedure to calculate the matrix $[S]$. For example, we want to determine the sensitivity of $p$ to the migration of the interface for the mode $i$. This field is denoted $D p\left[F_{i} \underline{n}\right.$. To calculate this field using the formulation developed in section 4.3, we first need to extend the velocity $F_{i} \underline{n}$ to the whole domain $\Omega$.

Let us define the extended velocity $\underline{\theta}_{i}$ as

$$
\underline{\theta}_{i}=F_{i}(s(\underline{x})) \underline{\nabla} \phi(\underline{x}) \mathbf{r}(\underline{x})
$$

Where the gradient of the level-set $\underline{\nabla} \phi$ is used to make the velocity normal to the interface. Since the level-set function is a signed distance function, it must be of norm 1 . The last function $\mathbf{r}(\underline{x})$ is a function which permits to extend the velocity $\Omega$. This function is only non zero in a thick band, where its value is 1 . This band can be defined as a layer of elements around the interface. The Figure 8 shows the band around a circular interface.

The size of the band determines the size on which the right side of (67) and (68) need to be computed. The optimal size of the band with regard to numerical accuracy will be the subject of further work. But, in all numerical experiments shown in this study, the band has a thickness of approximately 4 elements. As defined above, $s$ is a curvilinear coordinate along the interface $\Gamma$. This curvilinear coordinate needs to be extended in $\Omega$ for equation 87 to make sense. One possibility is to compute $s$ at each node $v$ of the mesh by taking the value of $s$ on the closest node to $v$ on the interface. This algorithm is very similar to some implementation of the velocity extension algorithm very common in level-set method, where a field only known on the iso-zero of the level-set need to be extended every where else [23].

Once the field $D p\left[\underline{\theta}_{i}\right]$ is known, we determine the modal projection of this field on the interface $\Gamma$. The coefficients found constitutes the vector $i$ of the matrix $[S]$. 

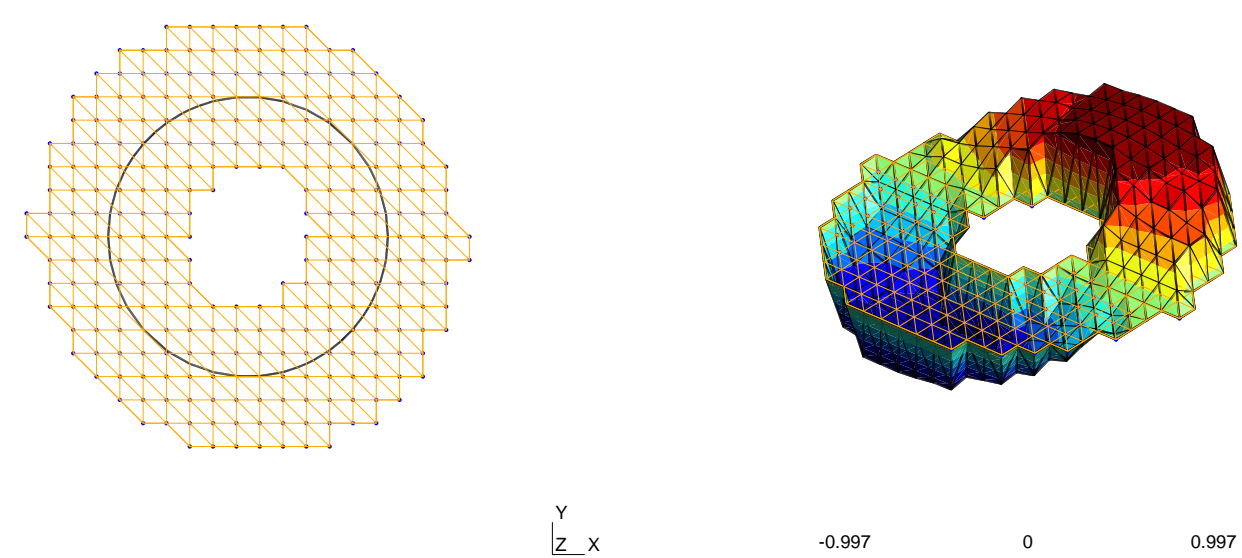

$-0.997$

0

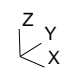

Figure 8: The band of elements around the level-set on the left, and the normal velocity on the right

Once the velocity field $\underline{v}_{k}$ is found, the final step is to propagate the level-set by solving the following Hamilton-Jacobi equation. [26] To solve it, we use the triangulated versions of the levelset methods as presented in [23], more preciselly, the so called explicit positive coefficient scheme.

$$
\frac{\partial \phi\left(\underline{x}, t^{*}\right)}{\partial t^{*}}+\left(\underline{v_{k}} \cdot \underline{n}\right)\left\|\nabla \phi\left(\underline{x}, t^{*}\right)\right\|=0
$$

Where $t^{*}$ designates a fictious time.

Once the level-set is propagated, we have to renormalize the level-set using the same type of equation.

Convergence criterion After each propagation step, and once the level-set is normalized, the equilibrium (50) is solved. Then, the following criteria is computed

$$
c_{p}=\left(\int_{\Gamma} p^{2} d \Gamma\right)^{\frac{1}{2}} \leq \varepsilon
$$

where $\varepsilon$ is a tolerance that can be set arbitrarily.

If the convergence criterion is not verfied, a new interface velocity is computed, and the levelset is once again propagated.

The whole algorithm, composed of two overlaped loops, is detailed below

\subsubsection{Discretization}

To enforce the constraint imposed on $\Omega_{c}$, we have used a Lagrange multiplier approach. We have to discretize both the displacement and the Lagrange multiplier fields. As explained in the previous part, we have to use specific mixed elements that satisfy the LBB condition. Unfortunately, the ridge function introduced in the X-FEM enrichment modify the finite dimensions subspace $V_{h}$. However, it is shown in [28] that the stability of enriched mixed element is preserved through the numerical inf-sup test. Where the pressure is defined (all elements that have at least one node inside $\Omega_{c}$ ), the element used is the $P 2 / P 1_{\text {cont }}$ (see Figure 9 ). This element is composed of a quadratic displacement and a linear continous pressure. Here, the use of continuous pressure element is possible unlike with the classical approach where this type of elements causes problems of 
convergence of the active-set algorithm. Other element are simply classical quadratic lagrange element for the displacement field. Each component of the displacement field is further enriched, at all the node $v e$ that have there support cut by $\Gamma$, by the function $F$ defined previously. This formulation therefore give a total number of degree of freedom $n d o f=2 * n v+1 * n v p+2 * n e+2 * n v e$ where $n v$ is the total number of nodes, $n e$ the total number of edges, $n v p$ the total number of node where a pressure is defined, and nve, the total number of node where the displacement field is enriched.

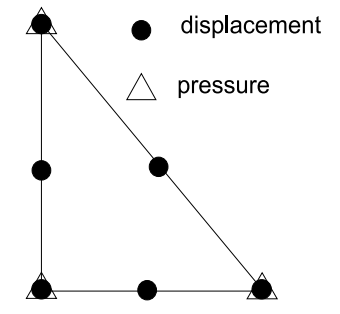

Figure 9: $P 2 / P 1_{\text {cont }}$ mixed element

\section{Numerical examples}

\subsection{Convergence studies}

Two cases will be studied here. A one-dimensional case and a cylinder case for which the analytical solution is known. The analytical solution will be used to determine the h-convergence of each method. To compare the exact and the numerical solution, we use the $L^{2}$ norm for the pressure field (90) and the energy norm for the displacement (91).

$$
\begin{aligned}
& e_{p}=\frac{\left(\int_{\Omega}\left(p_{h}-p_{e x}\right)^{2} d \Omega\right)^{\frac{1}{2}}}{\left(\int_{\Omega} p_{e x}^{2} d \Omega\right)^{\frac{1}{2}}} \\
& e_{e}=\frac{\left(\int_{\Omega}\left(\underline{\underline{(}}_{h}^{D}-\underline{\underline{\varepsilon}}_{e x}^{D}\right): \underline{\underline{\underline{C}}}:\left(\underline{\underline{\varepsilon}}_{h}^{D}-\underline{\underline{\varepsilon}}_{e x}^{D}\right) d \Omega\right)^{\frac{1}{2}}}{\left(\int_{\Omega} \underline{\underline{\varepsilon}}_{e x}^{D}: \underline{\underline{\underline{C}}}: \underline{\underline{\varepsilon}}_{e x}^{D} d \Omega\right)^{\frac{1}{2}}}
\end{aligned}
$$

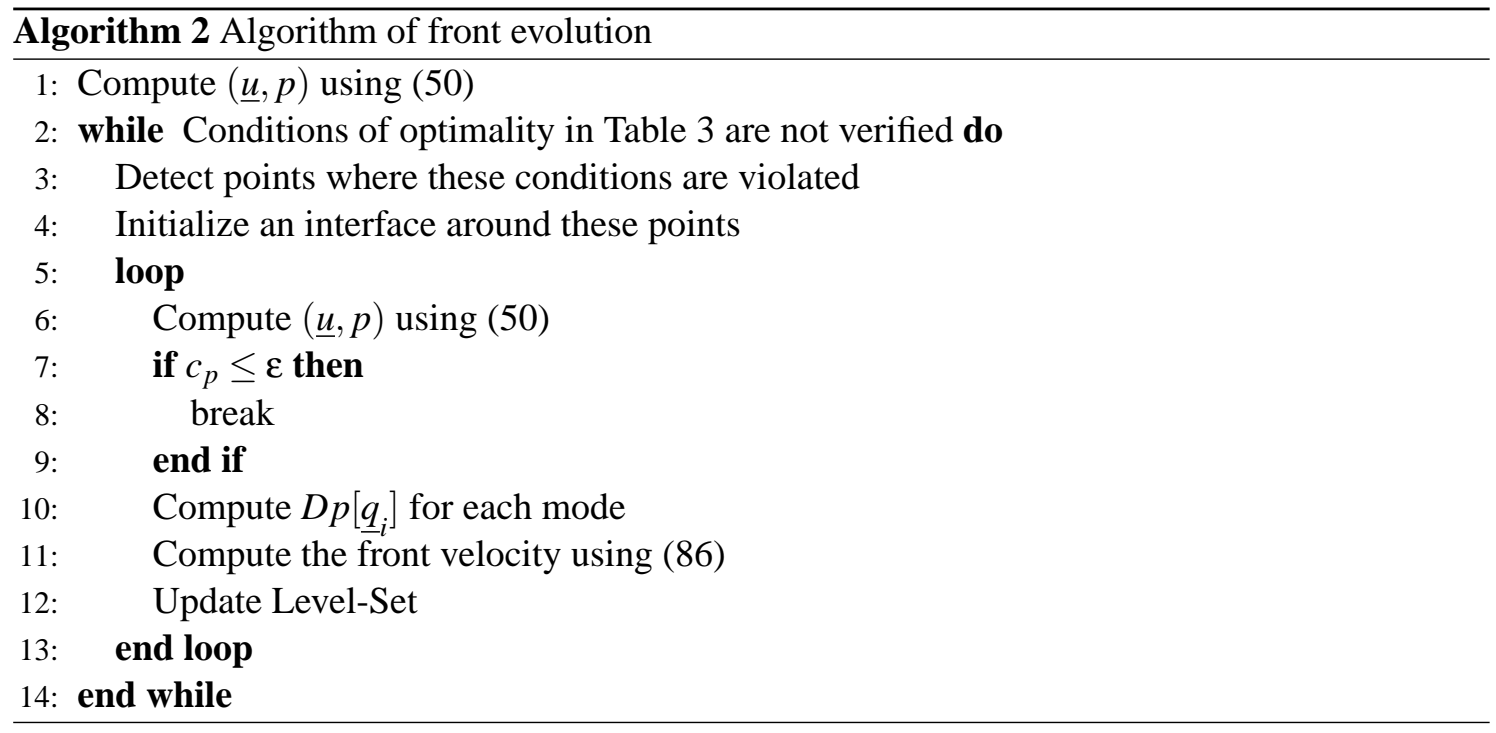




\subsubsection{One-dimensional case}

We apply now the new approach to solve the simple one-dimensional problem in the small strain elastostatic setting with uniaxial state of strain described in section 4.2. We wish to limit the uniaxial strain of a truss by an arbitrary value $\alpha$ under a prescribed load $f(x)=f x$. We set $\alpha=\frac{3}{8}$, $(\lambda+2 \mu)=1, f=1$ and $L=1$.

The analytical solution of this problem is given in Appendix B.
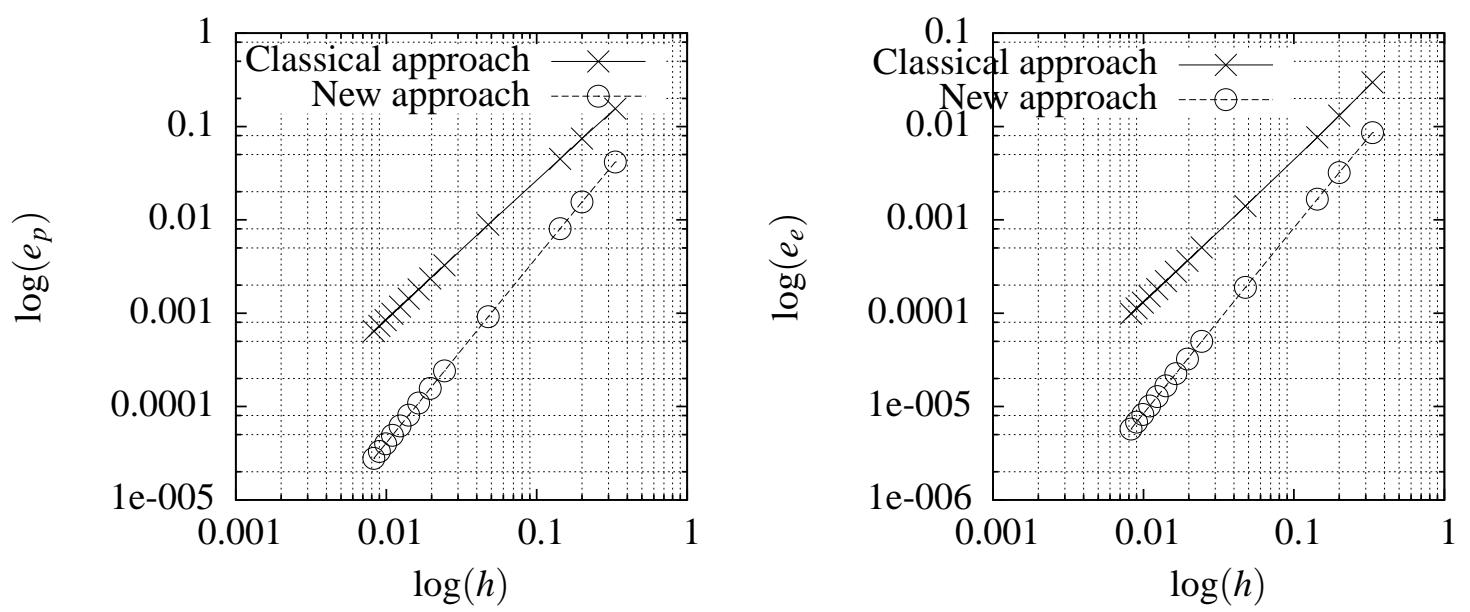

Figure 10: Convergence of the $L^{2}$ norm error on the pressure (left), and the energy norm error (right)

The results of convergence are presented in Figure 10. With the classical approach, the $L^{2}$ norm error on the pressure and the energy norm error on the displacement exhibits a rate of convergence in $O\left(h^{\frac{3}{2}}\right)$. The rate of convergence is degrated because of the presence of a singularity in the exact solution as we can see in Figure 6. With the new approach, thanks to the X-FEM enrichment, we can catch the singularity and we recover the optimal rate of convergence in $O\left(h^{2}\right)$.

\subsubsection{Two-dimensional case}

The second case studied here is an axisymmetric problem. It is presented in Figure 11. We consider a cylinder subjected to a volumetric force $\underline{f}=\underline{u}_{r}$, where $\underline{u}_{r}$ is the unit radial vector. The cylinder is held fixed at radius $a=0.5$ and the external radius is $b=1$. We choose $\lambda=0$ and $\mu=\frac{1}{2}$ for the Lamé coefficients. We want to limit the value of $\operatorname{Tr}(\underline{\underline{\varepsilon}})$ by $\alpha=0.5$. The analytical solution is given in Appendix C.

The results of convergence are presented in Figure 12. With the classical approach, the $L^{2}$ norm error on the pressure and the energy norm error on the displacement exhibits a rate of convergence in $O\left(h^{\frac{3}{2}}\right)$. As in the one-dimensional case, the rate of convergence is degraded because of the

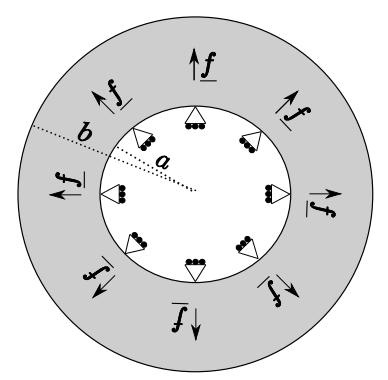

Figure 11: Axisymmetric problem 

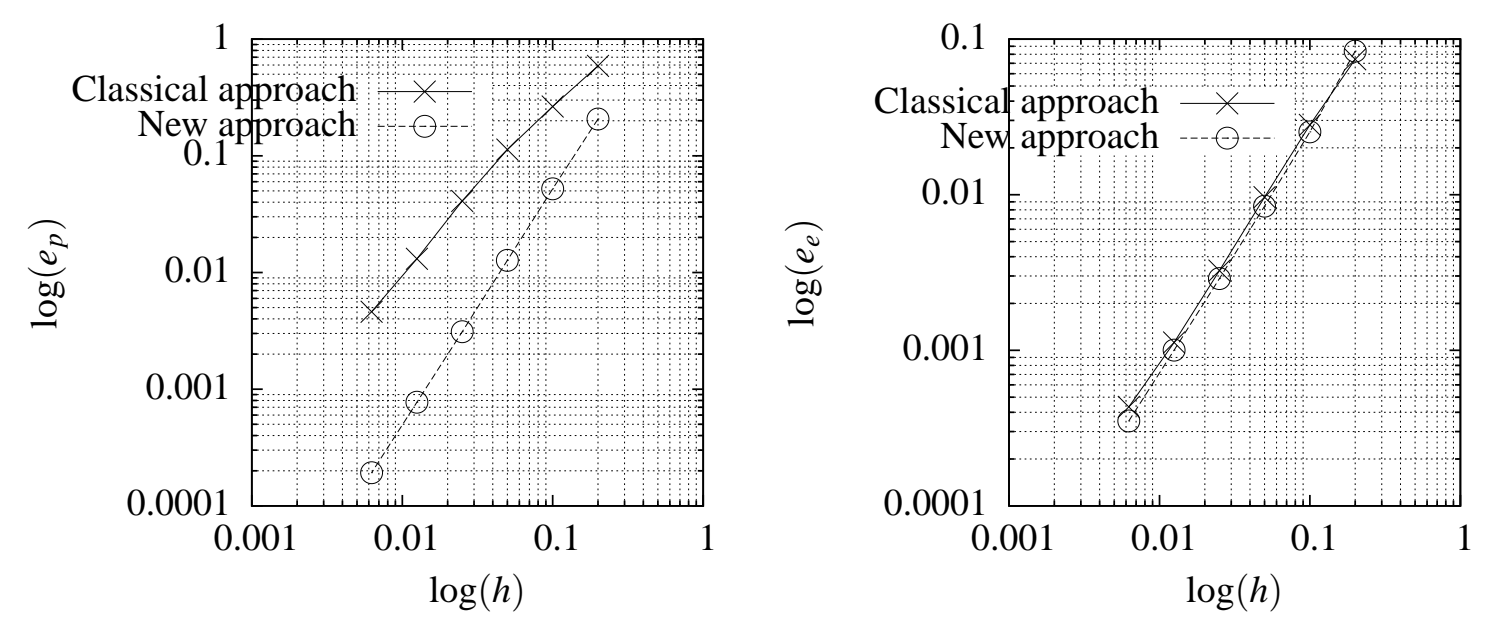

Figure 12: Convergence of the $L^{2}$ norm error on the pressure (left), and the energy norm error (right)

presence of a low regularity in the exact solution. With the new approach, thanks to the X-FEM enrichment, we can catch the low regularity and we recover the optimal rate of convergence in $O\left(h^{2}\right)$ for the $L^{2}$ norm error on the pressure. Unfortunately, we do not recover the optimal rate of convergence in $O\left(h^{2}\right)$ for the energy norm error on the displacement. It comes from the fact that the interface is discretized with straight edges while the displacement is quadratic. We could improve the convergence using the method described in [27].

\subsection{A more complex case and related numerical experiments.}

We want here to apply the new approach to a more complex two-dimensional case as shown in Figure 13. The example studied here is a zero centered 2D rectangular plate subjected to a body force $f(\underline{x})$ and held fixed at the bottom. The height $b$ of the plate is set to 1 and the width $a$ is set to 0.5 . We choose $\lambda=0.9$ and $\mu=0.6$ for the Lamé coefficients.

The following body force is chosen :

$$
\underline{f}(x, y, z)=x \underline{u}_{x}+y \underline{u}_{y}
$$

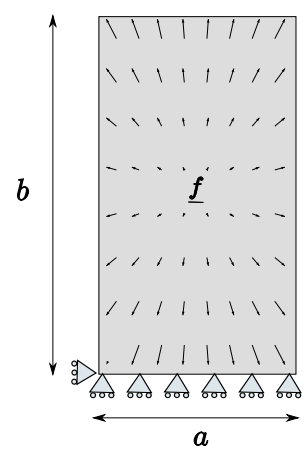

Figure 13: A more complex case

This kind of body force permits us to have a non homogeneous repartition of $\operatorname{Tr} \underline{\underline{\varepsilon}}$. Without any constraint, the equilibrium solution can be computed and in this case the iso-values of $\operatorname{Tr} \varepsilon$ are ellipses. With the constraint on, we do not know of an analytical solution for this example. It would be too complex to calculate the exact shape of $\Omega_{c}$, but this example exhibits an interesting repartition of $\operatorname{Tr} \underline{\underline{\varepsilon}}$ such that the numerical research of the shape of $\Omega_{c}$ involves several modes. 
The starting position of the interface $\Gamma$ is chosen circular and zero-centered. The initial radius of the cylinder is fixed arbitrarily at $r=0.1$. Only four modes are used to describe the shape changes. After four iterations, the convergence criteria is reached and the interface is close to an ellipse. It can be compared to the constrained zone as computed by the classical algorithm, by displaying the values of $\chi$. On figure 14 , left, we therefore show a representation of $\chi$, only evaluated at gauss point during the computation, as a linear by element field. On top of this field we display the iso-zero value of the level-set representing the interface as obtained with the new approach. As can be seen on the figure, both method give similar results in term of constrained zone. The new approach has the advantage of defining the zone in a more explicit sense.

The problem we are solving here are for now too small to do a proper efficency comparaison of the two methods: the solving time is below one minute on regular desktop for both method. The new method is probably, for equal meshes, more expensive at each iteration than the classical one. Indeed The sensibilities for each velocity mode need to be computed, and then an Hamilton Jacoby equation need to be solved. This last comment can still be nuanced a bit since the pressure field needed to enforce the equality constrain is only defined inside the constrained zone, delimited by the zero value of the level-set, and the Hamilton Jacoby equation could be solved in a narrow band only. Over all, the most expensive computations are the assembly and the factorisation of the linear system needed to solve the elasticity problem. For the mesh we used for the present test case, this cost is equivalent in both method, since the total number of degree of freedom is 11576 for the classical method and reach 10689 at convergence for the new method.

The advantages of the new method is that it is able to give more precise results on a given mesh than the classical one, as shown by the convergence rate in previous section. It provides a clear answer to the question of the position of the interface, which enable the enrichment its enrichment to better capture the irregularities of the fields and eventually the description of more involved physics at this interface.

In order to stress the presented algorithm a little bit more, we tried to solve the same problem with different starting position of the interface. Figure 14, middle and right, show the evolution of the constrained zone during the iterations. The middle picture show the evolution when the starting interface is an off center circle. The right picture show the evolution when the starting interface is made of two circles. In both cases the algorithm converge to the same final shape as the one obtain with the previously defeined starting interface. To obtain the correct results, more modes are needed in this two cases than for the first one. In fact this tests permitted to discovers that the numbers of mode used are optimally set when it is proportional to $l$, the total lenght of the interface divided by $h$, a characteristic size of the finite elements. Indeed, when the interface lenght is very small, there is no point to compute a large number of modes, since those modes are going to be very badly represented on the mesh. When the relative lenght of the interface become larger, more mode can be used. In the experiment shown here, we used a number of mode $n$ such as $n$ is the closest integer to $\frac{l}{2 h}$. Our numerical experiments also shown that, when during the iteration the local curvature of the interface become to high compared to the element size, the algorithm may take a very long time or even fail to converge. The case when we started with the interface made of two circle is one such a case. Indeed, when the two circles merge, there is a point where the local curvature could even become singular. This problem might be due to the fact that we neglicted the curvature term in equation 80 . To avoid computing the curvature, we used the following fix : as long as the residual in the newton method is big enough, the level-set function is smoothed prior to compute the new advance. We used a very simple smoothing the level-set : at each node $v$, the value of the level-set is replaced by the result of a least-square fit of the level-set values at each nodes connected to the current node $v$. 

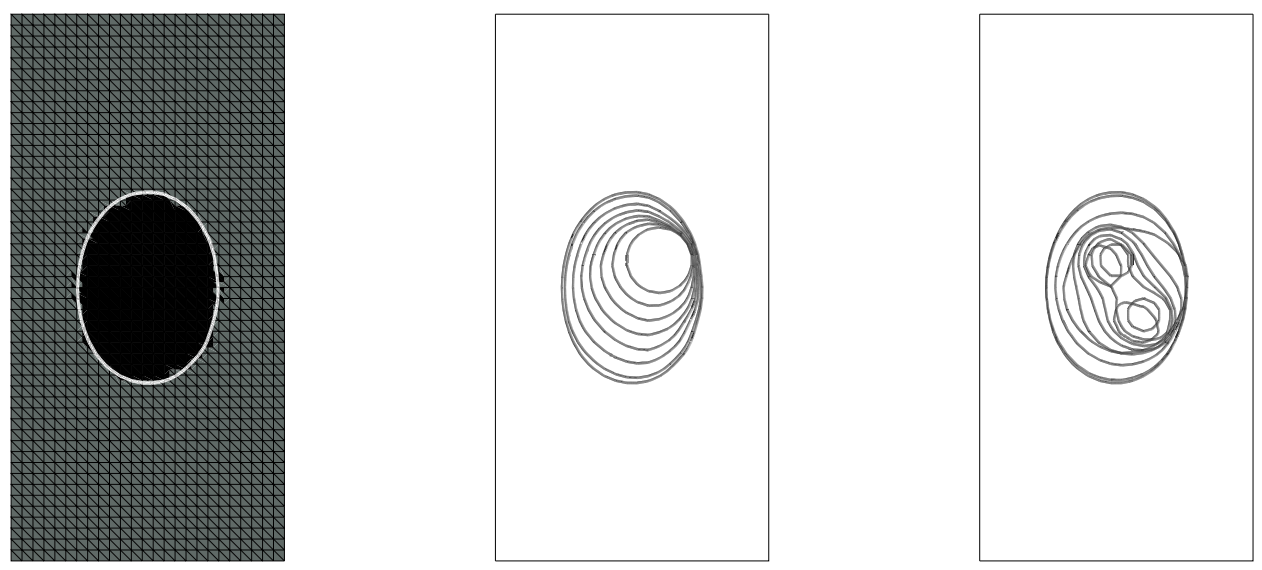

Figure 14: Left : Comparaison between the classical and new the method. In black, $\chi=1$, in grey $\chi=0$, for the classical method. The interface between constrained and unconstrained zone for the new method is the white line. Middle and Right : evolution of the interface during iterations for the new approach. Middle: the starting interface is one off centered circle. Right: the starting interface is made of two circle.

\section{Conclusion}

We have presented in this study a new approach to deal with continuum media subjected to an inequality constraint. The main difficulty of inequality constrained minimization problems is that the shape of the constrained zone is a priori unknown. Hence, the strategy proposed in this paper is to fix arbitrarily an initial constrained zone and to propagate it correctly until the correct shape is found following a specific criteria.

After a theoretical presentation of the problem and the classical approach, we have developed an algorithmic scheme that ensures to find the exact location of the interface. This new approach, relies on the notion of level-set, which is a powerful tool to represent moving interfaces in a domain, coupled with a X-FEM enrichment. Several numerical examples have been performed and exhibit a significant gain in convergence compared to the classical approach. Indeed the exact representation of the constrained zone avoids the loss of convergence due to the lack of regularity of the exact solution.

It must be made clear that proposed approach has some limitations related to the discretization. If the active zone is too small (or too thin), for the mesh size, it will not be detected. The detection will only be possible if the loading increases and the zone enlarges. If the knowledge of the loading for the active zone initiation is important, adaptivity could be introduced.

Finally, the new use of level sets to handle inequality contraints (or ILS in short for Inequality Level Set) introduced in this paper could be applied to other type of constraints such as inextensibility constraints in hyperelastic materials.

\section{Appendix A}

In this appendix, we demonstrate the equation (62). We consider the velocity $\underline{\theta}$ normal to the interface. If we take into account the continuity of the displacement field and normal stress accross the interface $\Gamma$ and assume the volumetric force $f$ to be continous accross $\Gamma$, we get

$$
\tau=\underline{n} \cdot[\underline{\underline{P}}] \cdot \underline{n}=\left(\psi_{f}-\psi_{c}\right)-(\underline{\underline{\sigma}} \cdot \underline{n}) \cdot\left(\left(\nabla \underline{u}_{c}-\underline{\nabla}_{f}\right) \cdot \underline{n}\right)
$$

We will next denote by $p_{c}$ the pressure field on the domain $\Omega_{c}$ and by $p_{f}$ the pressure field on the domain $\Omega_{f}$. We then have 


$$
p(\underline{x})=\left\{\begin{array}{rr}
p_{f}(\underline{x}) & \text { if } \underline{x} \in \Omega_{f} \\
p_{c}(\underline{x}) & \text { if } \underline{x} \in \Omega_{c}
\end{array}\right.
$$

In the same way, we denote by $\underline{\underline{\varepsilon}}_{c}$ the strain field on the domain $\Omega_{c}$ and by $\underline{\underline{\varepsilon}}_{f}$ the strain field on the domain $\Omega_{f}$ and we have

$$
\begin{gathered}
\underline{\underline{\varepsilon}}(\underline{x})= \begin{cases}\underline{\varepsilon}_{f}(\underline{x}) & \text { if } \underline{x} \in \Omega_{f} \\
\underline{\varepsilon}_{c}(\underline{x}) & \text { if } \underline{x} \in \Omega_{c}\end{cases} \\
\psi_{f}-\psi_{c}=\mu\left(\underline{\underline{\varepsilon}}_{c}: \underline{\underline{\varepsilon}}_{c}-\underline{\underline{\varepsilon}}_{f}: \underline{\underline{\varepsilon}}_{f}\right)+\frac{\lambda}{2}\left(\alpha^{2}-\left(\mathbf{T r} \underline{\underline{\varepsilon}}_{f}\right)^{2}\right) \\
=\mu\left(\underline{\underline{\varepsilon}}_{c}+\underline{\underline{\varepsilon}}_{f}\right):\left(\underline{\underline{\varepsilon}}_{c}-\underline{\underline{\varepsilon}}_{1}\right)+\frac{\lambda}{2}\left(\alpha+\operatorname{Tr} \underline{\underline{\varepsilon}}_{f}\right)\left(\alpha-\mathbf{T r}_{\underline{\underline{\varepsilon}}}\right) \\
=\left(\mu\left(\underline{\underline{\varepsilon}}_{c}+\underline{\underline{\varepsilon}}_{f}\right)+\frac{\lambda}{2}\left(\alpha+\mathbf{T r} \underline{\underline{\varepsilon}}_{f}\right) \underline{\underline{I}}\right):\left(\underline{\underline{\varepsilon}}_{c}-\underline{\underline{\varepsilon}}_{f}\right) \\
=\left(\frac{\underline{\underline{\sigma}}_{l}+\underline{\underline{\sigma}}_{c}}{2}-\frac{p_{c}}{2} \underline{\underline{I}}\right):\left(\underline{\underline{\varepsilon}}_{c}-\underline{\underline{\varepsilon}}_{f}\right)
\end{gathered}
$$

We thus get

$$
\begin{aligned}
\tau=\underline{n}[\underline{\underline{P}}] \underline{\underline{n}} & =\left(\frac{\underline{\underline{\sigma}}_{l}+\underline{\underline{\sigma}}_{c}}{2}\right):\left(\underline{\underline{\varepsilon}}_{c}-\underline{\underline{\varepsilon}}_{f}\right)-(\underline{\underline{\sigma}} \cdot \underline{n}) \cdot\left(\left(\nabla \underline{u}_{c}-\nabla \underline{u}_{f}\right) \cdot \underline{n}\right) \\
& =\frac{p_{c}}{2}\left(\underline{\operatorname{Tr}}_{f}-\alpha\right) \\
& =(\lambda+2 \mu)^{-1} \frac{p_{c}^{2}}{2} \\
& =\frac{(\lambda+2 \mu)}{2}\left(\operatorname{Tr}_{\underline{\underline{\varepsilon}}}-\alpha\right)^{2}
\end{aligned}
$$

On the intertface, we have due to the continuity of the normal stress vector

$$
p=\left(\kappa+\frac{3}{4} \mu\right)\left(\operatorname{Tr} \underline{\underline{\varepsilon}}_{f}-\alpha\right)=(\lambda+2 \mu)\left(\operatorname{Tr}_{\underline{\varepsilon}}-\alpha\right)
$$

\section{Appendix B}

In this appendix, we detail the analytical solution for the $1 \mathrm{D}$ problem

$$
\begin{gathered}
u(x)=\left\{\begin{array}{r}
\alpha x \quad \text { if } 0 \leq x \leq y \\
\frac{f}{6(\lambda+2 \mu)}\left(y^{3}-x^{3}\right)+\frac{f}{2(\lambda+2 \mu)} L^{2}(x-y)+\alpha y \quad \text { if } L \geq x \geq y
\end{array}\right. \\
\varepsilon(x)=\left\{\begin{aligned}
\alpha & \text { if } 0 \leq x \leq y \\
\frac{f}{2(\lambda+2 \mu)}\left(L^{2}-x^{2}\right) & \text { if } L \geq x \geq y
\end{aligned}\right. \\
p(x)=\left\{\begin{aligned}
\frac{f}{2}\left(L^{2}-x^{2}\right)-(\lambda+2 \mu) \alpha & 0 \leq x \leq y \\
0 & \text { if } L \geq x \geq y
\end{aligned}\right.
\end{gathered}
$$

Let us now compute the potential energy $\Phi$ in the system for any given location $y$ of the interface. 


$$
\begin{aligned}
\Phi= & \int_{0}^{y} \frac{(\lambda+2 \mu) \alpha^{2}}{2} d x+\int_{y}^{L} f^{2} \frac{\left(L^{2}-x^{2}\right)^{2}}{8(\lambda+2 \mu)} d x-\int_{0}^{y} f \alpha x^{2} d x- \\
& -\int_{y}^{L} f x\left(\frac{f}{6(\lambda+2 \mu)}\left(y^{3}-x^{3}\right)+\frac{f}{2(\lambda+2 \mu)} L^{2}(x-y)+\alpha y\right) d x
\end{aligned}
$$

Denoting $\xi=\frac{y}{L}$, and after calculus, we obtain

$$
\begin{aligned}
\Phi(\xi) & =\frac{f^{2} L^{5}}{(\lambda+2 \mu)}\left(\frac{\xi^{5}}{40}-\frac{\xi^{3}}{48}+\frac{\xi}{128}-\frac{1}{15}\right) \\
\frac{\partial \Phi}{\partial \xi} & =\frac{f^{2} L^{5}}{(\lambda+2 \mu)}\left(\frac{\xi^{4}}{8}-\frac{\xi^{2}}{16}+\frac{1}{128}\right)=L \tau(y)
\end{aligned}
$$

where $\tau(y)=\left[[P]_{y}\right.$ is the configurational force at the interface $y$.

\section{Appendix C}

In this appendix, we detail the analytical solution for the axisymmetric problem

$$
\begin{gathered}
p(r)=\left\{\begin{array}{rr}
-r+D & \text { if } r<r_{e} \\
0 & \text { if } r \geq r_{e}
\end{array}\right. \\
\underline{u}=u(r) \underline{u}_{r}
\end{gathered}
$$

with

$$
\begin{aligned}
& u(r)=\left\{\begin{aligned}
\alpha \frac{r}{2}+\frac{C}{r} & \text { if } r<r_{e} \\
-\frac{r^{2}}{3}+\frac{C 1 r}{2}+\frac{C 2}{r} & \text { if } r \geq r_{e}
\end{aligned}\right. \\
& \underline{\underline{\varepsilon}}=\left(\begin{array}{ccc}
\varepsilon_{r r} & 0 & 0 \\
0 & \varepsilon_{\theta \theta} & 0 \\
0 & 0 & 0
\end{array}\right) \\
& \varepsilon_{r r}=\left\{\begin{aligned}
\frac{\alpha}{2}-\frac{C}{r^{2}} & \text { if } r<r_{e} \\
-\frac{2}{3} r+\frac{C 1}{2}-\frac{C 2}{r^{2}} & \text { if } r \geq r_{e}
\end{aligned}\right. \\
& \varepsilon_{\theta \theta}=\left\{\begin{aligned}
\frac{\alpha}{2}+\frac{C}{r^{2}} & \text { if } r<r_{e} \\
-\frac{r}{3}+\frac{C 1}{2}+\frac{C 2}{r^{2}} & \text { if } r \geq r_{e}
\end{aligned}\right.
\end{aligned}
$$

where $r$ designates the radial coordinate. And $D$ is the a constant defined by 


$$
\begin{array}{r}
D=-\frac{2}{3} r_{e}+\frac{C_{1}}{2}-\frac{C_{2}}{r_{e}^{2}}-\frac{\alpha}{2}+\frac{C}{r_{e}^{2}}+r_{e} \\
C=-\frac{\alpha a^{2}}{2} \\
C_{1}=\left(\frac{\alpha}{3}+\frac{\alpha}{2}+C+\frac{r_{e}^{3}}{3}\right) \frac{2}{1+r_{e}^{2}} \\
C_{2}=-\frac{2}{3}+\frac{C_{1}}{2}
\end{array}
$$

The constant $r_{e}$ is the zero of the equation $g(y)=0$, with $g$

$$
g(y)=-\frac{2}{3} y+\frac{C_{1}}{2}-\frac{C_{2}}{y^{2}}-\frac{\alpha}{2}+\frac{C}{y^{2}}
$$

\section{Bibliography}

\section{References}

[1] Computational Contact and Impact Mechanics: Fundamentals of Modeling Interfacial Phenomena in Nonlinear Finite Element Analysis, T. A. Laursen, Springer-Verlag, Heidelberg, 2002.

[2] Contact problems in elasticity: a study of variational inequalities and finite element methods, N. Kikuchi , J. T. Oden, SIAM Publication, Philadelphia, 1988.

[3] A mixed formulation for frictional contact problems prone to Newton like solution methods, $\mathrm{P}$. Alart, A. Curnier, Computer Methods in Applied Mechanics and Engineering, vol. 92, 353375, 1991.

[4] Modeling holes and inclusions by level sets in the extended finite element method, N. Sukumar, D.L. Chopp, N. Moës, T. Belytschko, Computer Methods in Applied Mechanics and Engineering, vol. 190, 6183-6200, 2001.

[5] A computational approach to handle complex microstructure geometries, N. Moës, M. Cloirec , P. Cartraud, J. Remacle, Computer Methods in Applied Mechanics and Engineering, vol. 192, 3163-3177, 2003.

[6] Problèmes de contact frottant en grandes transformations : du continu au discret, H. Ben Dhia, I. Vautier, M. Zarroug, Revue Européenne des Element Finis, vol. 9, 1-3, 243-261, 2000.

[7] Computational Contact Mechanics, P. Wriggers, J. Wiley and Sons, New-York, 2002.

[8] A generalized Newton method for contact problems with friction, A. Curnier, P. Alart, Journal de Mécanique Théorique et Appliquée, Special Issue, supplement 1 to vol. 7, 67-82, 1988.

[9] La méthode des contraintes actives appliquée au contact unilatéral, G. Dumont, Note Interne EDF, HI- 75/93/016, 1993.

[10] Algorithme des contraintes actives et contact unilatéral sans frottement, G. Dumont, Revue Européenne des Éléments Finis, vol. 4, 55-73, 1995.

[11] Les inéquations en mécaniques et en physique, G. Duvaut, J.L. Lions, Dunod, 1972. 
[12] Augmented Lagrangian and operator-splitting methods in nonlinear mechanics, R. Glowinski, P. Le Tallec, SIAM, Philadelphia, 1989.

[13] Large deformation frictional contact mechanics: continuum formulation and augmented Lagrangian treatment, G. Pietrzak, A. Curnier, Computer Methods in Applied Mechanics and Engineering, vol. 177, 351-381, 1999.

[14] Une formulation pour traiter le contact frottement en $3 d$ dans Code Aster, H. Ben Dhia, I. Vautier, Rapport EDF, HI- 75/99/007/A, 1999.

[15] Hybrid frictional contact particles-in elements, H. Ben Dhia, M. Zarroug, Revue Européenne des Éléments Finis, vol. 9, 2-4, 417-430, 2002.

[16] Eléments mixtes de contact frottant en grandes transformations et applications, M.Zarroug, Thèse de doctorat, École Centrale de Paris, 2002.

[17] Contribution à la modélisation mécanique et numérique des problèmes de contact-impact, C. Zammali, Thèse de doctorat, École Centrale de Paris, 2005.

[18] Approche X-FEM pour la fissuration sous contact des structures industrielles, S. Geniaut, Thèse de doctorat, Ecole Centrale de Nantes, 2006.

[19] Mixed and Hybrid Finite Element Methods, F. Brezzi, M. Fortin, Springer, New York, 1991.

[20] Mechanical Transformations and discontinuities along a moving surface, R.-M. PradeillesDuval and C.Stolz, Journal of the Mechanics and Physics of Solids, Vol. 43, 1, 91-121, 1995

[21] The elastic energy-momentum tensor, J.D. Eshelby, Journal of Elasticity, 5(3-4), 321-335, 1975.

[22] Fronts propagating with curvature dependent speed: algorithms based on hamilton-jacobi formulations, S. Osher, J. Sethian, Journal of Computational Physics, vol. 79, 12-49, 1988.

[23] Level Set Methods and Fast Marching Methods : Evolving Interfaces in Computational Geometry, Fluid Mechanics, Computer Vision, and Materials science J. Sethian, Cambridge University Press, 1999.

[24] A finite element method for crack growth without remeshing, N. Moës, J. Dolbow, T. Belytschko, International Journal for Numerical Methods in Engineering, vol. 46, 131-150, 1999.

[25] Shape sensitivity analysis in linear elastic fracture mechanics, E. Taroco, Computer Methods in Applied Mechanics and Engineering, vol. 188, 697-712, 2000.

[26] Non planar $3 d$ crack growth by the extended finite element and level-sets. part ii : level set update., A. Gravouil, N. Moës, and T. Belytschko, International Journal for Numerical Methods in Engineering, vol. 53 ,2569-2586, 2002.

[27] Studied X-FEM enrichment to handle material interfaces with higher order finite element, K. Dréau, N. Chevaugeon, N. Moës, Computer Methods in Applied Mechanics and Engineering, vol. 199, 29-32, 1922-1936, 2010.

[28] Stability of incompressible formulations enriched with X-FEM, G. Legrain, N. Moës, A. Huerta, Computer Methods in Applied Mechanics and Engineering, vol. 197, 1835-1849, 2007.

[29] A level set based model for damage growth: the thick level set approach, N. Moës, C. Stolz, N. Chevaugeon, P.-E. Bernard, International Journal for Numerical Methods in Engineering, 2010. 